Thesis Number:

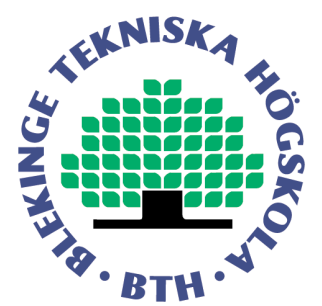

\title{
Fuzzy Logic and Approximate Reasoning
}

\begin{abstract}
Abdul Khaliq and Amais Ahmad
This thesis is presented as part of Degree of Master of Sciences in Mathematical Modelling and Simulation

Blekinge Institute of Technology

2010
\end{abstract}

School of Engineering

Department of Mathematics and Sciences

Blekinge Institute of Technology, Sweden

Supervisor: Elisabeth Rakus-Andersson

Examiner: Elisabeth Rakus-Andersson 


\section{Contact Information:}

\section{Author:}

Abdul Khaliq and Amais Ahmad

email: khaliqsyed@gmail.com, amaisahmad@gmail.com

\section{Supervisor:}

Elisabeth Rakus-Andersson

Department of Mathematics and Sciences

School of Engineering, BTH

Blekinge Institute of Technology, Sweden

email: elisabeth.andersson@bth.se

\section{Examiner:}

Elisabeth Rakus-Andersson

Department of Mathematics and Sciences

School of Engineering, BTH

Blekinge Institute of Technology, Sweden

email: elisabeth.andersson@bth.se 


\section{Abstract}

Two of the most exemplary capabilities of the human mind are the capability of using perceptions (human knowledge) in purposeful ways and the capability of approximating perceptions by statements in natural language. Understanding these capabilities and emulating them by linguistic approximation is the crux of our thesis. There has been a rapid growth in the number and variety of applications of fuzzy logic. In a narrow sense, fuzzy logic is a logical system which is an extension of multivalued logic and is intended to serve as logic of approximate reasoning. But in a wider sense, fuzzy logic is more or less synonymous with the theory of fuzzy sets. In classical logic the propositional value of a statement is either true (1) or false (0) but in lukasiewicz logic we gave value as a truthfulness to a certain proposition between $[0,1]$. As a generalization of many valued logic, fuzzy logic was established in order to deal with those fuzzy propositions and to underlie approximate reasoning. We have calculated the fuzzy truth values and compare the results of different operations (conjunction, disjunction etc) with the approach to Baldwin's (1979) and with the help of modus ponens law (If $\mathrm{p} \rightarrow \mathrm{q}$ and $p^{\prime}$ then $q^{\prime}$.).

There are many chemical reactions that are very sensitive and a little change in temperature and particle size can create serious problems. We have developed the idea of approximate reasoning and fuzzy logic to find the approximate value of reaction rate with the given conditions by means of the extended modus ponens law. The methodology is very simple and can be applied to several other chemical reactions in the similar way by connecting AND and OR operations. The result $Q^{\prime}$ can be found by the fuzzy relation equation $Q^{\prime}=P^{\prime}$ o $\mathrm{R}$ where "o" is the max-min composition of $P^{\prime}$ and R operation. Result $Q^{\prime}$ for the certain situation is in the form of fuzzy set, in which we choose the value with maximum membership degree. 


\section{Acknowledgements}

We would like to thank to our dear ALLAH who gave us this ability and chance to understand and learn the in-depth knowledge of science and technology at Blekinge Institute of Technology, BTH. We would like to show our gratitude to our honorable thesis supervisor Prof. Dr.Elisabeth Rakus Andersson for her guidance, feedback and support throughout our thesis work. We will also express our deepest gratitude to all the teachers who let us understand the real aspect of mathematics, and for letting us learn more about Mathematical Modelling and simulation. We would like to thank our program manager Dr.Raisa Khamitova for giving us his valuable time in sorting out issues related with our subjects and assignments.

Last but not least, we also express our deepest gratitude to our beloved parents, for always having encouraged us and supported us in every possible way throughout our studies both financially and moral. 



\section{Contents}

$\begin{array}{ll}\text { Abstract } & \text { iii }\end{array}$

Acknowledgements $\quad$ V

List of Figures $\quad$ xi

1 Classical Logic 1

1.1 Logic . . . . . . . . . . . . . . . . . . . . . . 1

1.1.1 Examples of Logic . . . . . . . . . . . . . . . . . . . . . . . . . . . . . . . . . .

1.1.2 Logic on Higher Level . . . . . . . . . . . . . . . . . . . . 2

1.2 Classical Logic . . . . . . . . . . . . . . . . . . . . 3

1.2.1 Symbols Used in Classical Logic . . . . . . . . . . . . . . . 3

1.2.2 Basic Operations on Classical Truth Values . . . . . . . . . 3

1.2.3 Truth Table . . . . . . . . . . . . . . . . . . 4

1.2.4 Example of Basic Classical Logical Operations . . . . . . . 4

2 Lukasiewicz Logic $\mathbf{5}$

2.13 -Valued Lukasiewicz Logic . . . . . . . . . . . . . . 5

2.1.1 Operations on 3-Valued Lukasiewicz Truth Expressions . . 6

2.1.2 Truth Table for 3-Valued Lukasiewicz Logic . . . . . . . . 6

2.1.3 Example of Basic Operations in 3-valued Lukasiewicz Logic 6

2.1.4 The Guiding Principles of the System of 3-valued Lukasiewicz Logic . . . . . . . . . . . . . . . . . . . . . . . . . .

2.1.5 Model Operators . . . . . . . . . . . . . . . 7

2.1.6 Example of Model Operators . . . . . . . . . . . . . 8

2.1.7 Modalities in Classical Logic . . . . . . . . . . . . . . 8

2.1.8 Comparison of 3-valued Lukasiewicz Logic and Classical Logic . . . . . . . . . . . . . . . . 9 9

2.2 Many-valued Generalization of the 3-Valued Lukasiewicz Logic . . 9

2.2.1 Division of Unit Interval . . . . . . . . . . . . . . . . . . . 9

2.2.2 4-valued Lukasiewicz Logic . . . . . . . . . . . . . . . . . . 10

2.2.3 Example of 4-valued Lukasiewicz Logic . . . . . . . . . . . 11 
2.2.4 Example of Comparison between Classical and Lukasiewicz Logic . . . . . . . . . . . . . . . . . . 11

2.2.5 Example of many-valued Lukasiewicz Logic . . . . . . . . . 13

3 Fuzzy Logic 15

3.1 Introduction . . . . . . . . . . . . . . . . 15

3.1 Fuzzy Set . . . . . . . . . . . . . . . 15

3.1.2 Example of Fuzzy Set . . . . . . . . . . . . . . . . . . . 15

3.2 Distinguished Features of Fuzzy Logic . . . . . . . . . . . . . . . 16

3.3 The Truth Value Set of Fuzzy Logic . . . . . . . . . . . . . . . . . 17

3.3.1 Comparison of Linguistic Fuzzy Truth Values and Numerical Lukasiewicz Truth Values . . . . . . . . . . . . . 18

3.3.2 Operations on Fuzzy Truth Values . . . . . . . . . . . 18

3.3.3 Extension Principle for Fuzzy Sets . . . . . . . . . . . . . 18

3.4 Use of Extension Principle for the Operations on Fuzzy Truth Values 21

3.4 .1 Negation . . . . . . . . . . . . . . . . . 22

3.4.2 Example of Negation . . . . . . . . . . . . . . . . . . 22

3.4.3 Conjunction . . . . . . . . . . . . . . . . . 22

3.4.4 Example of Conjunction . . . . . . . . . . . . . 23

3.4 .5 Disjunction ........................ 23

3.4.6 Example of Disjunction . . . . . . . . . . . . . . 23

3.4 .7 Implication . . . . . . . . . . . . . . . . . . . . 24

3.4 .8 Example of Implication . . . . . . . . . . . . . . . . . . . . 24

3.4 .9 Equivalence . . . . . . . . . . . . . . . . . 25

3.4.10 Example of Equivalence . . . . . . . . . . . . . . . . 25

3.5 Baldwin Approach to Fuzzy Truth Values . . . . . . . . . . . . 26

3.5.1 Fuzzy Truth Value Sets in Baldwin Approach . . . . . . . 26

3.5.2 Negations in Baldwin Approach . . . . . . . . . . . . . 27

3.5.3 Graphical Baldwin Approach to Fuzzy Truth Values . . . . 28

3.6 Linguistic Approximation . . . . . . . . . . . . . . . . . . 28

3.6.1 Example of Linguistic Approximation . . . . . . . . . . . . 29

3.7 Modus Ponens Law with Fuzzy truth-values . . . . . . . . . . . . 29

4 Approximate Reasoning 35

4.1 Classical IF-THEN Rule . . . . . . . . . . . . . . . . . . . . 36

4.1.1 Example of Classical IF-THEN Rule . . . . . . . . . . . . . 36

4.2 Fuzzy IF-THEN Rule . . . . . . . . . . . . . . . . . . . . 36

4.2.1 Example of Linguistic Fuzzy sets . . . . . . . . . . . . . 37

4.3 Models for Approximations . . . . . . . . . . . . . . . . . 37

4.4 Schematic Procedure . . . . . . . . . . . . . . . . 38

4.5 Basic Rules of Inference . . . . . . . . . . . . . . . . . . . . . . . . . . . . 38

4.5.1 Example of Modus Ponens . . . . . . . . . . . . . . 39

4.5.2 Example of Modus Tollens . . . . . . . . . . . . . . . . . 39 
4.6 Generalized Modus Ponens or Fuzzy Rule of Inference . . . . . . . 40

4.7 Rules of Finding Fuzzy Relation R . . . . . . . . . . . . . . . . . . 41

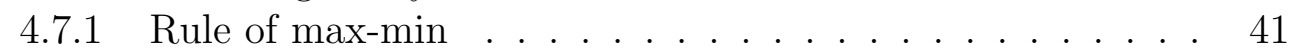

4.7 .2 Binary Rule . . . . . . . . . . . . . . . . . . 41

4.7 .3 Lukasiewicz Rule . . . . . . . . . . . . . . . . . . . . 41

4.7 .4 Min-Rule . . . . . . . . . . . . . . . . . . 41

4.7.5 Example of Finding Fuzzy Relation R . . . . . . . . . . . . 42

4.7.6 Example of Fuzzy Modus Ponens . . . . . . . . . . . . . . 43

4.7.7 Conjunction Form of the Antecedent . . . . . . . . . . . 44

5 Approximate Reasoning in Chemical Reactions 47

6 News 53

6.1 Latest News . . . . . . . . . . . . . . . . . . . . . . 54

$\begin{array}{lll}7 & \text { Conclusion } & 57\end{array}$

$\begin{array}{ll}\text { Bibliography } & 59\end{array}$ 



\section{List of Figures}

2.1 Comparison of classical and Lukasiewicz logic . . . . . . . . . . . 12

3.1 Fuzzy set of apartment rent . . . . . . . . . . . . . . 16

3.2 Baldwin approach to fuzzy truth values . . . . . . . . . . . . . 28

4.1 Fuzzy sets of temperature domain . . . . . . . . . . . . . 36

4.2 Linguistic fuzzy sets . . . . . . . . . . . . . . . . 37 



\section{Chapter 1}

\section{Classical Logic}

\subsection{Logic}

We all give reasons in our daily life. We try to figure out what is so, reasoning on the basis of what we already know. We try to persuade others that something is so by giving them reasons. Logic is the study of what counts as a good reason for what, and why. It is all about reasons. Every day we consider possibilities, we think about what follows from different alternatives and we weigh up competing positions or options. To understand good reasoning we must have an idea of the kinds of things we reason about. Logic concerns itself with reasons for believing something instead of something else.

In everyday situations arguments are dialogues between people. In logic we do not study all of the features of these dialogues. We concentrate on the propositions people express when they give reasons for things. For us an argument is a list of propositions, called the premises, followed by a word such as 'therefore' or 'so' and then another proposition called the conclusion [1].

"Logic is the study of valid arguments. It is a systematic approach to distinguish valid arguments from invalid arguments. It is an instinctive art. It has one or more premises and a conclusion. In advancing an argument one claim that premise or premises that support the decision or give any help to readers to make conclusion."

\subsubsection{Examples of Logic}

\section{Example 1}

1. Rome is the capital of Italy, and this plane lands in Rome; so the plane lands in Italy. 
2. Moscow is the capital of the USA; so you can't go to Moscow without going to the USA.

Conclusion: The first piece of reasoning is fine; but second is pretty hopeless, and does not give any one with an elementary knowledge of geography. The second premise is simply false.

\section{Example 2}

1. $\mathrm{x}<\mathrm{y}$

2. $\mathrm{y}$ is a small value.

Conclusion: Therefore ' $\mathrm{x}$ ' is also a small value.

\section{Example 3}

1. David is a man.

2. All men are tall.

Conclusion: Therefore David is tall.

In each case the claim before 'so' is called premise and the claim after is called conclusion.

\subsubsection{Logic on Higher Level}

On higher level, Logic is a science which developed out of the self awareness of thinkers. Logic is of value to all individuals bettering their daily reasoning processes and thus their efficacy in dealing with their lives and their work. It helps you to arrive at the solution of problems more rapidly and efficiently.

Logic teaches us to pursue and verify knowledge. It is based on an acknowledgement of the possibility of human error but also implies our ability to correct errors where veracity or falsity is hard to establish. It tells us at least how reasonable or forced our judgments are. Logic is concerned with the formalities of reasoning without so much regard to its subject matter.

\section{Example}

1. A is a president of the United States.

2. A is a son of the president of the United States.

Conclusion: Therefore, there is someone who is both a president of the United States and a son of a president of the United States. 


\subsection{Classical Logic}

Classical logic is the simplest of all major logics. Classical logic is logic in which there are only two truth values for a statement or proposition, i.e, there are only two possible values that we can assign to certain statements.

The truth values are

1. True $(1$, yes $)$

2. False $(0, \mathrm{No})$

A proposition can be true or false but not both at the same time.

\section{For example}

"sun rises in the east" is a true statement and has truth value 1.

Normally we use 1's and 0's in mathematical classical logic and true, false in propositional classical logic.

\subsubsection{Symbols Used in Classical Logic}

We use following symbols to connect two truth values in different ways.

$\neg$ is used for Negation (NOT).

$\wedge$ is used for Conjunction (AND).

$\checkmark$ is used for Disjunction (OR).

$\rightarrow$ is used for an Implication sign.

$\leftrightarrow$ is used for Equivalence.

These are the basic notations and all other notations used in classical and other logics are derived from these notations.

\subsubsection{Basic Operations on Classical Truth Values}

Operations on propositional classical logic can be described in terms of tables of 0 's and 1's called truth tables. Truth tables for the classical logic are based upon the following basic operations.

1. $\neg \mathrm{p}=1-\mathrm{p}$

2. $\mathrm{p} \vee \mathrm{q}=\max (\mathrm{p}, \mathrm{q})$

3. $\mathrm{p} \wedge \mathrm{q}=\min (\mathrm{p}, \mathrm{q})$

4. $\mathrm{p} \rightarrow \mathrm{q}=\min \{1,1-\mathrm{p}+\mathrm{q}\}$

where $\mathrm{p}$ and $\mathrm{q}$ are two propositions and their truthfulness is used as inputs in the following truth table. 


\subsubsection{Truth Table}

\begin{tabular}{|cc|c|c|c|c|}
\hline $\mathrm{p}$ & $\mathrm{q}$ & $\neg \mathrm{p}$ & $\mathrm{p} \vee \mathrm{q}$ & $\mathrm{p} \wedge \mathrm{q}$ & $\mathrm{p} \rightarrow \mathrm{q}$ \\
\hline 0 & 0 & 1 & 0 & 0 & 1 \\
\hline 0 & 1 & 1 & 1 & 0 & 1 \\
\hline 1 & 0 & 0 & 1 & 0 & 0 \\
\hline 1 & 1 & 0 & 1 & 1 & 1 \\
\hline
\end{tabular}

\subsubsection{Example of Basic Classical Logical Operations}

Let us consider two propositions

$$
\begin{aligned}
& \mathrm{p}=\text { "We are sitting in a restaurant" } \\
& \mathrm{q}=\text { "We are taking tea" }
\end{aligned}
$$

Now according to above operations and truth table we have

1. $\neg \mathrm{p}=$ "We are not sitting in a restaurant"

$\neg \mathrm{q}=$ "We are not taking tea

2. $\mathrm{p} \vee \mathrm{q}=$ "We are sitting in a restaurant or we are taking tea"

which means that the compound statement is true when one of the p and $q$ or both $\mathrm{p}$ and $\mathrm{q}$ will be true.

3. $\mathrm{p} \wedge \mathrm{q}=$ "We are sitting in a restaurant and we are taking tea"

which means that the compound statement is true only when both $\mathrm{p}$ and $\mathrm{q}$ will be true.

4. $\mathrm{p} \rightarrow \mathrm{q}=$ "If we are sitting in a restaurant then we are taking tea"

which means that the compound statement will be wrong when true statement implies the wrong one. We can also say that $\mathrm{p}$ is a sufficient condition for $\mathrm{q}$ or $\mathrm{q}$ is a necessary condition for $\mathrm{p}$.

There should be a relation between premise and conclusion in the implication, e.g, If I fall into the lake then I will get wet.

We also use implication in theorems,

e.g, If $\mathrm{ABC}$ is the right triangle with right angle at $\mathrm{B}$ then $A C^{2}=A B^{2}+B C^{2}$. 


\section{Chapter 2}

\section{Lukasiewicz Logic}

Classical logic works well for mathematical proofs, but it does not describe how we reason about most nonmathematical matters.

For example:

"I will wear a t- shirt tomorrow" (or)

"I will not wear a t-shirt tomorrow."

Classical logic says one of those is already true. So is my free will just an illusion? We need a logic that can say 'maybe'.

"Lukasiewicz logic is a non-classical, multi-valued logic in which we assign a certain value as a truthfulness of a given statement on an interval [0,1]."

In 1920 Lukasiewicz proposed the theory of three valued logic which was generalized later on to $\mathrm{n}$-valued $(\mathrm{n}=2,3, \ldots .$.$) logic.$

\section{$2.1 \quad 3$-Valued Lukasiewicz Logic}

In 3-valued Lukasiewicz logic we have an intermediate state between "true" and "false" which can be interpreted as "may be true" or "may be false" with numeric value of $1 / 2$.

\section{Example}

I will be in America in 2 months......................True (1)

And I will not be in America in 2 months............False (0)

I May be in America in 2 months................. May be $(1 / 2)$ 


\subsubsection{Operations on 3-Valued Lukasiewicz Truth Expres- sions}

$\mathrm{P}$ is any statement about which we have to make conclusions whether it is true $(\mathrm{T})$, false $(\mathrm{F})$ or indeterminate $(\mathrm{I})$. And $\mathrm{v}(\mathrm{p})$ is a truth value of statement $\mathrm{p}$ which could be 1,0 or $1 / 2$.

We introduce the following operations for two primitive statements $\mathrm{p}$ and $\mathrm{q}$ due to Lukasiewicz's suggestions to sample their results in the truth table.

1. $\mathrm{v}(\neg \mathrm{p})=1-\mathrm{v}(\mathrm{p})$

2. $\mathrm{v}(\mathrm{p} \vee \mathrm{q})=\max (\mathrm{v}(\mathrm{p}), \mathrm{v}(\mathrm{q}))=\mathrm{v}(\mathrm{p} \cup \mathrm{q})=\mathrm{v}(\mathrm{p}) \vee \mathrm{v}(\mathrm{q})$

3. $\mathrm{v}(\mathrm{p} \wedge \mathrm{q})=\min (\mathrm{v}(\mathrm{p}), \mathrm{v}(\mathrm{q}))=\mathrm{v}(\mathrm{p} \cap \mathrm{q})=\mathrm{v}(\mathrm{p}) \wedge \mathrm{v}(\mathrm{q})$

4. $\mathrm{v}(\mathrm{p} \rightarrow \mathrm{q})=1 \wedge[1-\mathrm{v}(\mathrm{p})+\mathrm{v}(\mathrm{q})]=\min [1,1-\mathrm{v}(\mathrm{p})+\mathrm{v}(\mathrm{q})]$

5. $\mathrm{v}(\mathrm{p} \leftrightarrow \mathrm{q})=\min [\mathrm{v}(\mathrm{p} \rightarrow \mathrm{q}), \mathrm{v}(\mathrm{q} \rightarrow \mathrm{p})]=\mathrm{v}(\mathrm{p} \rightarrow \mathrm{q}) \wedge \mathrm{v}(\mathrm{q} \rightarrow \mathrm{p})$

\subsubsection{Truth Table for 3-Valued Lukasiewicz Logic}

\begin{tabular}{|c|cc|ccc|ccc|ccc|ccc|}
\hline $\mathrm{p}$ & $\mathrm{q} / \mathrm{p}$ & $\neg \mathrm{p}$ & \multicolumn{2}{c|}{$\mathrm{p} \wedge$} & $\mathrm{q}$ & $\mathrm{p} \vee$ & $\mathrm{q}$ & $\mathrm{p} \rightarrow$ & $\mathrm{q}$ & $\mathrm{p}$ & $\leftrightarrow$ & $\mathrm{q}$ \\
& & & 1 & $\frac{1}{2}$ & 0 & 1 & $\frac{1}{2}$ & 0 & 1 & $\frac{1}{2}$ & 0 & 1 & $\frac{1}{2}$ & 0 \\
\hline 1 & 1 & 0 & 1 & $\frac{1}{2}$ & 0 & 1 & 1 & 1 & 1 & $\frac{1}{2}$ & 0 & 1 & $\frac{1}{2}$ & 0 \\
\hline$\frac{1}{2}$ & $\frac{1}{2}$ & $\frac{1}{2}$ & $\frac{1}{2}$ & $\frac{1}{2}$ & 0 & 1 & $\frac{1}{2}$ & $\frac{1}{2}$ & 1 & 1 & $\frac{1}{2}$ & $\frac{1}{2}$ & 1 & $\frac{1}{2}$ \\
\hline 0 & 0 & 1 & 0 & 0 & 0 & 1 & $\frac{1}{2}$ & 0 & 1 & 1 & 1 & 0 & $\frac{1}{2}$ & 1 \\
\hline
\end{tabular}

where $\mathrm{p}$ and $\mathrm{q}$ are truth values of two different statements and $\mathrm{q} / \mathrm{p}$ is the value of $q$ on condition that $p$. This table is constructed by combining each value of $p$ with all three values of $q$. This can be illustrated by the following example.

\subsubsection{Example of Basic Operations in 3-valued Lukasiewicz Logic}

Let us take two values of $p$ and $q$

$\mathrm{p}=1 / 2$ and $\mathrm{q}=1$

so we see the second row in this case.

$\neg p=1-1 / 2=1 / 2$

which is shown on the last position in second column. 
$\mathrm{p} \wedge \mathrm{q}=\min (1 / 2,1)=1 / 2$

which is shown on the first position in third column.

$\mathrm{p} \vee \mathrm{q}=\max (1 / 2,1)=1$

which can be seen on the first position in fourth column.

$\mathrm{p} \rightarrow \mathrm{q}=\min (1,1-1 / 2+1)=\min (1,3 / 2)=1$

which is shown on the first position in fifth column.

$\mathrm{p} \leftrightarrow \mathrm{q}=\min (\mathrm{p} \rightarrow \mathrm{q}, \mathrm{q} \rightarrow \mathrm{p})$

where $\mathrm{q} \rightarrow \mathrm{p}=\min (1,1-1+1 / 2)=\min (1,1 / 2)=1 / 2$

So

$\mathrm{p} \leftrightarrow \mathrm{q}=\min (1,1 / 2)=1 / 2$

shown on the first position in last column.

Similarly we can check the results for any other combination of values of $\mathrm{p}$ and q.

\subsubsection{The Guiding Principles of the System of 3-valued Lukasiewicz Logic}

1. There are to be three truth values ( $\mathrm{T}, \mathrm{I}, \mathrm{F}$ or $1,1 / 2,0)$ so ordered in term of decreasing truthfulness.

2. The negation of a statement of given truth values is its opposite in truthfulness.

3. The truth value of a conjunction is the falsest and of a disjunction the truest of the truth values of its components.

4. The truth value of $\mathrm{p} \rightarrow \mathrm{q}$ is the same as that of $\neg p \vee q$ except the truth value corresponding to $\mathrm{I} \rightarrow \mathrm{I}$.

5. The truth value of $\mathrm{p} \leftrightarrow \mathrm{q}$ is to be the same as that of $(\mathrm{p} \rightarrow \mathrm{q}) \wedge(\mathrm{q} \rightarrow \mathrm{p})$.

\subsubsection{Model Operators}

Lukasiewicz implemented the idea of Aristotelian logic that proposition regarding future contingent matters have a truth status that does not corresponds to either of the orthodox truth values of truth and falsity. In carrying out this idea one must introduce corresponding complications into the truth rules for propositional connectives. Lukasiewicz solution to this problem consists of truth tables for any proposition or statement [2].

With a view to the future contingency interpretation of the third truth value 
(I), Lukasiewicz introduced model operators of possibility and necessity (symbolically $\diamond a n d \square$ ) into his 3 valued logic. These are to be subject of the truth table presented below.

\begin{tabular}{|c|c|c|}
\hline $\mathrm{v}(\mathrm{p})$ & $\diamond \mathrm{p}$ & $\square \mathrm{p}$ \\
\hline 1 & 1 & 1 \\
\hline $1 / 2$ & 1 & 0 \\
\hline 0 & 0 & 0 \\
\hline
\end{tabular}

Thus symbolically $\diamond \mathrm{p}$ is to be true if $\mathrm{p}$ is either true or intermediate but is false if $\mathrm{p}$ is definitely false.

\subsubsection{Example of Model Operators}

Let us consider the future prediction about rain, i.e. Tomorrow will be rain.

Now if this is true $(\mathrm{v}(\mathrm{p})=1)$, then there is possibility of rain tomorrow $(\diamond \mathrm{p}$ $=1)$ and also it is necessary $(\square \mathrm{p}=1)$ to be true. Otherwise the values $\mathrm{v}(\mathrm{p})$ and $\square$ p will contradict.

If it is partially true that tomorrow will be rain $(\mathrm{v}(\mathrm{p})=1 / 2)$, then there is still possibility of rain $(\diamond \mathrm{p}=1)$ but is not necessary $(\square \mathrm{p}=0)$ to be true that tomorrow will be rain.

If it is false that tomorrow will be rain $(\mathrm{v}(\mathrm{p})=0)$, then there is not any possibility of rain $(\diamond \mathrm{p}=0)$ and also not necessary $(\square \mathrm{p}=0)$ to be true that tomorrow will be rain.

\subsubsection{Modalities in Classical Logic}

Truth functional treatment of modalities was not possible in two valued logic. The closest we can come to specifying modalities in classical logic is

\begin{tabular}{|c|c|c|}
\hline $\mathrm{v}(\mathrm{p})$ & $\diamond \mathrm{p}$ & $\square \mathrm{p}$ \\
\hline 1 & 1 & 1 \\
\hline 0 & 0 & 0 \\
\hline
\end{tabular}

And then $\mathrm{v}(\mathrm{p}) \equiv \diamond p$ and $v(p) \equiv \square \mathrm{p}$

So $\square p \equiv \diamond \mathrm{p}$ will all be logical truths so that model distinctions collapse in 3 valued logic however we will have the desirable implications $\mathrm{p} \rightarrow \diamond p$ and $\square p \rightarrow$ $v(p)$ and $\square p \rightarrow \diamond p$ without being saddled with their undesirable converses so that model distinctions are preserved. 


\subsubsection{Comparison of 3-valued Lukasiewicz Logic and Clas- sical Logic}

Three valued truth table agrees with the two valued ones when only T's(1's) and F's (0's) are involved as we can see in truth table of 3-valued Lukasiewicz logic. i.e.

\begin{tabular}{|c|cc|ccc|ccc|ccc|ccc|}
\hline $\mathrm{p}$ & $\mathrm{q} / \mathrm{p}$ & $\neg \mathrm{p}$ & \multicolumn{2}{c|}{$\mathrm{p} \wedge$} & $\mathrm{q}$ & $\mathrm{p} \vee$ & $\mathrm{q}$ & $\mathrm{p} \rightarrow$ & $\mathrm{q}$ & $\mathrm{p}$ & $\leftrightarrow$ & $\mathrm{q}$ \\
& & & 1 & $\frac{1}{2}$ & 0 & 1 & $\frac{1}{2}$ & 0 & 1 & $\frac{1}{2}$ & 0 & 1 & $\frac{1}{2}$ & 0 \\
\hline 1 & 1 & 0 & 1 & $\frac{1}{2}$ & 0 & 1 & 1 & 1 & 1 & $\frac{1}{2}$ & 0 & 1 & $\frac{1}{2}$ & 0 \\
\hline$\frac{1}{2}$ & $\frac{1}{2}$ & $\frac{1}{2}$ & $\frac{1}{2}$ & $\frac{1}{2}$ & 0 & 1 & $\frac{1}{2}$ & $\frac{1}{2}$ & 1 & 1 & $\frac{1}{2}$ & $\frac{1}{2}$ & 1 & $\frac{1}{2}$ \\
\hline 0 & 0 & 1 & 0 & 0 & 0 & 1 & $\frac{1}{2}$ & 0 & 1 & 1 & 1 & 0 & $\frac{1}{2}$ & 1 \\
\hline
\end{tabular}

Lukasiewicz used this 3-Valued Logic as a base to generalize it into n-valued Lukasiewicz Logic.

\subsection{Many-valued Generalization of the 3-Valued Lukasiewicz Logic}

It is a logic in which we assign a certain value to a truthfulness of given statement on an interval $[0,1]$ as in classical logic we assign ' 0 ' for false statement and ' 1 ' for true statement. But sometimes we come across many kinds of true and false statements like true, very true, very very true, rather false in our everyday life. Truth-values are real numbers between 0 and 1 for the proposition (say p) and is denoted by

$$
v(p) \in[0,1]
$$

"As Lukasiewicz generalized his 3-valued logic to many-valued logic so the operations on truth expressions in this case are the same as in 3-valued logic case."

\subsubsection{Division of Unit Interval}

Let us divide the interval from 0 to 1 by inserting evenly spaced division points for a total of $n$ points $(n \geq 2)$. 


\begin{tabular}{|c|c|c|}
\hline $\mathrm{n}$ & Division & Division Points (Truth Values) \\
\hline 2 & $1 / 1,0 / 1$ & $(1,0)$ \\
\hline 3 & $2 / 2,1 / 2,0 / 2$ & $(1,1 / 2,0)$ \\
\hline 4 & $3 / 3,2 / 3,1 / 3,0 / 3$ & $(1,2 / 3,1 / 3,0)$ \\
$:$ & $:$ & $:$ \\
$:$ & $:$ & $:$ \\
$:$ & $:$ & $:$ \\
$\mathrm{n}$ & $1=(\mathrm{n}-1) /(\mathrm{n}-1),(\mathrm{n}-2) /(\mathrm{n}-1)$, & \\
& $\ldots, 2 / \mathrm{n}-1,1 / \mathrm{n}-1,0 / \mathrm{n}-1=0$ & \\
\hline
\end{tabular}

Given the propositional connectives based on the arithmetical operations and taking the members of this series as truth-values, we obtain the series Ln of Lukasiewicz many-valued logic. It is readily seen that:

1. $L_{2}$ is identical with two valued classical logic.

2. $L_{3}$ is identical with 3 -valued system of Lukasiewicz.

So the system of the series will be many valued generalizations both of the classical two valued system (L2) and of the Lukasiewicz (L3).

Moreover one can take the further possibility of obtaining two infinite valued systems as follows

1. For the system $L_{s 0}$ we take 0 and 1 together with all the rational numbers (i.e all fractions $\mathrm{n} / \mathrm{m}$ ) between 0 and 1 as truth values.

2. For the system $L_{s 1}$ we take all the real numbers from the interval 0 to 1 as truth values.

So we can construct truth table $L_{n}$ for any value of $\mathrm{n}$.

\subsubsection{4-valued Lukasiewicz Logic}

Let us consider $\mathrm{n}=4$, then for $L_{4}$ the truth table is as follows

\begin{tabular}{|c|c|c|c|c|c|c|}
\hline $\mathrm{p}$ & $q / p$ & $\neg \mathrm{p}$ & $\mathrm{p} \wedge \mathrm{q}$ & $p \vee q$ & $p \rightarrow q$ & $\mathrm{p} \leftrightarrow \mathrm{q}$ \\
\hline & & & $\begin{array}{llll}1 & \frac{2}{3} & \frac{1}{3} & 0\end{array}$ & $\begin{array}{llll}1 & \frac{2}{3} & \frac{1}{3} & 0\end{array}$ & $\begin{array}{llll}1 & \frac{2}{3} & \frac{1}{3} & 0\end{array}$ & $\begin{array}{llll}1 & \frac{2}{3} & \frac{1}{3} & 0\end{array}$ \\
\hline 1 & 1 & 0 & $\begin{array}{llll}1 & \frac{2}{3} & \frac{1}{3} & 0\end{array}$ & $\begin{array}{llll}1 & 1 & 1 & 1 \\
\end{array}$ & $\begin{array}{llll}1 & \frac{2}{3} & \frac{1}{3} & 0\end{array}$ & $\begin{array}{llll}1 & \frac{2}{3} & \frac{1}{3} & 0\end{array}$ \\
\hline$\frac{2}{3}$ & $\frac{2}{3}$ & $\frac{1}{3}$ & $\begin{array}{llll}\frac{2}{3} & \frac{2}{3} & \frac{1}{3} & 0\end{array}$ & $\begin{array}{llll}1 & \frac{2}{3} & \frac{2}{3} & \frac{2}{3} \\
\end{array}$ & $\begin{array}{llll}1 & 1 & \frac{2}{3} & \frac{1}{3} \\
\end{array}$ & $\begin{array}{llll}\frac{2}{3} & 1 & \frac{2}{3} & \frac{1}{3}\end{array}$ \\
\hline$\frac{1}{3}$ & $\frac{1}{3}$ & $\frac{2}{3}$ & $\begin{array}{llll}\frac{1}{3} & \frac{1}{3} & \frac{1}{3} & 0\end{array}$ & $\begin{array}{llll}1 & \frac{2}{3} & \frac{1}{3} & \frac{1}{3} \\
\end{array}$ & $\begin{array}{llll}1 & 1 & 1 & \frac{2}{3} \\
\end{array}$ & $\frac{1}{3} \quad \frac{2}{3}$ \\
\hline 0 & 0 & 1 & $\begin{array}{lllll}0 & 0 & 0 & 0 \\
\end{array}$ & $\begin{array}{llll}1 & \frac{2}{3} & \frac{1}{3} & 0\end{array}$ & $\begin{array}{llll}1 & 1 & 1 & 1 \\
\end{array}$ & $\begin{array}{llll}0 & \frac{1}{3} & \frac{2}{3} & 1\end{array}$ \\
\hline
\end{tabular}


where $p$ and $q$ are truth values of two different statements and $q / p$ is the value of $\mathrm{q}$ on the condition that $\mathrm{p}$. This table is constructed by combining each value of $\mathrm{p}$ with all four values of $\mathrm{q}$. This can be illustrated by the following example.

\subsubsection{Example of 4-valued Lukasiewicz Logic}

Let us take two values of $\mathrm{p}$ and $\mathrm{q}$

$\mathrm{p}=2 / 3$ and $\mathrm{q}=1 / 3$

so we see the second row in this case.

$\neg \mathrm{p}=1-2 / 3=1 / 3$

$\neg \mathrm{q}=1-1 / 3=2 / 3$

$\mathrm{p} \wedge \mathrm{q}=\min (2 / 3,1 / 3)=1 / 3$

we can see on the third position in third column.

$\mathrm{p} \vee \mathrm{q}=\max (2 / 3,1 / 3)=2 / 3$

we can see on the third position in fourth column.

$\mathrm{p} \rightarrow \mathrm{q}=\min (1,1-2 / 3+1 / 3)=2 / 3$

we can see on the third position in fifth column.

$\mathrm{p} \leftrightarrow \mathrm{q}=\min (\mathrm{p} \rightarrow \mathrm{q}, \mathrm{q} \rightarrow \mathrm{p})$

where

$\mathrm{q} \rightarrow \mathrm{p}=\min (1,1-1 / 3+2 / 3)=1$

so

$\mathrm{p} \leftrightarrow \mathrm{q}=\min (2 / 3,1)=2 / 3$

we can see this value on the third position in last column.

We can check the results for the other combinations of the values of $p$ and $q$ in a similar way.

Similarly we can construct any other truth table for different values of $\mathrm{n}$ in $L_{n}$.

\subsubsection{Example of Comparison between Classical and Lukasiewicz Logic}

Let us consider $\mathrm{p}=$ "appreciated customers" in a a local bank, which is depended upon the amount of money (SEK) on their accounts. 
Following the data of customer financial situations we evaluate the truth value of "appreciated position" in the bank by using classical and Lukasiewicz logic.

\begin{tabular}{|c|c|c|c|}
\hline Account No. & Deposit in millions & $\begin{array}{c}\mathrm{v}(\mathrm{p}) \text { in classical } \\
\text { two-valued logic }\end{array}$ & $\begin{array}{c}\mathrm{v}(\mathrm{p}) \text { in Lukasiewicz } \\
\text { multi-valued logic }\end{array}$ \\
\hline $001-78$ & 100 & 1 & 1 \\
\hline $167-36$ & 93 & 1 & 0.98 \\
\hline $475-84$ & 79 & 1 & 0.80 \\
\hline $368-02$ & 71 & 1 & 0.78 \\
\hline $745-45$ & 54 & 1 & 0.65 \\
\hline $745-34$ & 41 & 0 & 0.39 \\
\hline $730-17$ & 33 & 0 & 0.24 \\
\hline $904-47$ & 27 & 0 & 0.15 \\
\hline $397-01$ & 18 & 0 & 0.09 \\
\hline $376-33$ & 7 & 0 & 0.01 \\
\hline
\end{tabular}

The following graph shows the difference between truth values belonging to different logical systems.

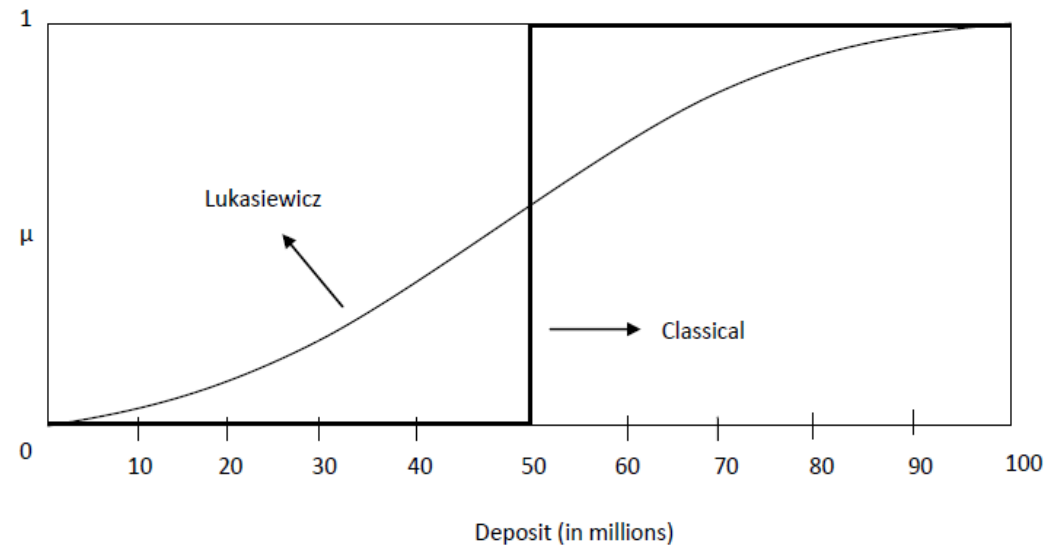

Figure 2.1: Comparison of classical and Lukasiewicz logic 


\subsubsection{Example of many-valued Lukasiewicz Logic}

Let $\mathrm{p}$ and $\mathrm{q}$ be the statements given as

$\mathrm{p}=$ "water level in a damm is high"

$\mathrm{q}=$ "it is raining"

Classical logic is failed here because it gives only two values for each of the above statements that water level is high [True(1)] or water level is not high [False(0)]. Similarly, it is raining [True(1)] or it is not raining [False(0)]. But in this case water level is high and it is raining but we don't know how much the level is high and how much intense the rain is? i.e, what is the truthfulness of the above statements $\mathrm{p}$ and $\mathrm{q}$. That's why we use Lukasiewicz logic to precise the given information by assigning the value according to the truthfulness. Suppose that the statement ' $p$ ' is true in the following sense.

$\mathrm{v}(\mathrm{p})=1$

i.e water level is very high.

$\mathrm{v}(\mathrm{p})=0.9$

i.e water level is high.

$\mathrm{v}(\mathrm{p})=0.7$

i.e. water level is rather high.

$\mathrm{v}(\mathrm{p})=0.5$

i.e. water level is moderately high.

Similarly, we can assign values to the statement q according to the reality.

Now take any two values of both statements.

"water level is rather high" with $\mathrm{v}(\mathrm{p})=0.7$

"it is raining heavily" with $\mathrm{v}(\mathrm{p})=0.9$

1. $\mathrm{v}(\neg p)=$ "water level is not rather high" $=1-\mathrm{v}(\mathrm{p})=1-0.7=0.3$

$\mathrm{v}(\neg q)=$ "it is not raining heavily" $=1-\mathrm{v}(\mathrm{q})=1-0.9=0.1$

2. $\mathrm{v}(\mathrm{p} \vee \mathrm{q})=$ "water level is rather high or it is raining heavily"

$\mathrm{v}(\mathrm{p} \vee \mathrm{q})=\max [\mathrm{v}(\mathrm{p}), \mathrm{v}(\mathrm{q})]=\max [0.7,0.9]=0.9$

3. $\mathrm{v}(\mathrm{p} \wedge \mathrm{q})=$ "water level is rather high and it is raining heavily" $\mathrm{v}(\mathrm{p} \wedge \mathrm{q})=\min [\mathrm{v}(\mathrm{p}), \mathrm{v}(\mathrm{q})]=\min [0.7,0.9]=0.7$

4. $\mathrm{v}(\mathrm{p} \rightarrow \mathrm{q})=$ "if water level is rather high then it is raining heavily" $\mathrm{v}(\mathrm{p} \rightarrow \mathrm{q})=\min [1,1-\mathrm{v}(\mathrm{p})+\mathrm{v}(\mathrm{q})]=\min [1,1-0.7+0.9]=\min [1,1.2]=1$ 



\section{Chapter 3}

\section{Fuzzy Logic}

\subsection{Introduction}

Fuzzy Logic is a logic in which the truth values are fuzzy subsets of the unit interval with linguistic labels such as

true, false, not true, very true, quite true, not very true and not very false etc.

The main difference between Lukasiewicz logic and fuzzy logic is that, in Lukasiewicz logic truth value for certain statement or proposition is a single value in $[0,1]$, but in fuzzy logic the truth value is fuzzy set of the unit interval.

\subsubsection{Fuzzy Set}

In classical set theory, elements fully belong to set or are fully excluded. But a fuzzy set $\mathrm{A}$ in universe $\mathrm{X}$ is the set whose elements belong to $\mathrm{X}$ partially also. The grade of belonging of each element is determined by a membership function $\mu_{A}$ given by

$$
\mu_{A}: X \rightarrow[0,1], \text { where } \mathrm{x} \in \mathrm{X}
$$

A finite fuzzy set can be denoted as

$$
\mathrm{A}=\mu_{A}\left(x_{1}\right) / x_{1}+\mu_{A}\left(x_{2}\right) / x_{2}+\ldots+\mu_{A}\left(x_{n}\right) / x_{n}
$$

\subsubsection{Example of Fuzzy Set}

Let us consider the rent for student apartment. We assume that below $\$ 100$ the rent is not expensive with membership degree 0, and above $\$ 250$ it is too expensive with membership degree 1. Between these two values, an increasing membership degree can be seen. It is not essential that the membership of rent linearly increase. 


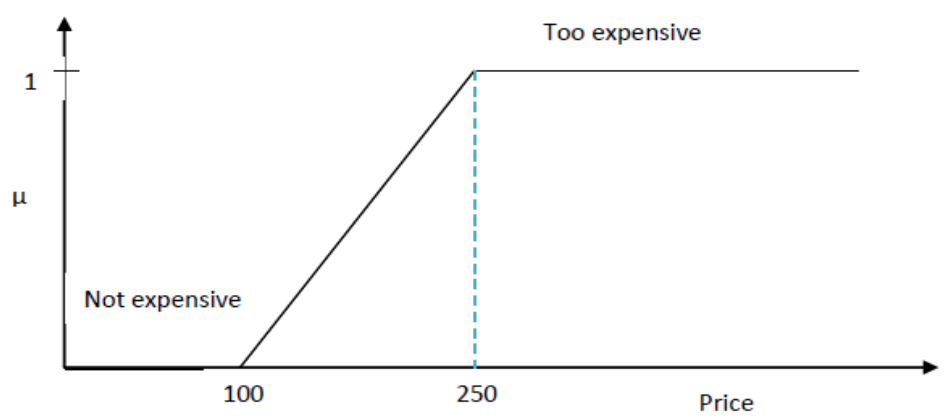

Figure 3.1: Fuzzy set of apartment rent

\subsection{Distinguished Features of Fuzzy Logic}

1. Fuzzy truth values are expressed in linguistic terms.

2. Imprecise truth tables.

3. Rules of inference where validity is approximate rather than exact.

In these aspects fuzzy logic differs significantly from standard logical systems ranging from the classical Aristotelian logic to inductive logics and many valued logics with set truth-values. Fuzzy logic is fuzzy extension of a non-fuzzy multivalued logic [3] which constitutes a base logic for fuzzy logic. In fuzzy logic we actually assign the membership degree to a statement according to the degree of truthfulness in the interval $[0,1]$.

It is convenient to use standard Lukasiewicz logic (which we have explained in first chapter) as a base logic for fuzzy logic . 


\subsection{The Truth Value Set of Fuzzy Logic}

The truth value set ' $\mathrm{T}$ ' of fuzzy logic is assumed to be generated by a context-free grammar with a semantic rule providing a means of computing the meaning of each linguistic truth value ' $\tau$ ' in ' $\mathrm{T}$ ' as a fuzzy subset of $[0,1]$. It is a countable set of the form

$\mathrm{T}=\{$ true, false not true, very true, not very true, more or less true, rather true, not very true, not very false,..., etc $\}$

Each element of this set represents a fuzzy subset of the truth value set of Lukasiewicz logic i.e. $[0,1]$. Thus the meaning of a linguistic truth value ${ }^{\prime} \tau^{\prime}$ in ' $\mathrm{T}$ ' is assumed to be a fuzzy subset of $[0,1]$, more specifically let [4]

$$
\mu_{\tau}:[0,1] \rightarrow[0,1] \text { denote the membership function of ' } \tau^{\prime} .
$$

Then the meaning of ' $\tau^{\prime}$ as a fuzzy subset of $[0,1]$ is expressed by

$$
\tau=\int_{0}^{1} \mu_{\tau}(v) / v
$$

where the integral sign denotes the union of fuzzy singletons $\mu_{\tau}(v) / v$, signifying that the membership of the numerical truth value ' $v$ ' with the linguistic truth value $\tau$ is $\mu_{\tau}(\mathrm{v})$ or the grade of membership of $\mathrm{v}$ in the fuzzy set labeled $\tau$ is $\mu_{\tau}$ (v).

If the support of $\tau$ is a finite subset $\left\{v_{1}, v_{2}, \ldots, v_{n}\right\}$ of $[0,1], \tau$ may be expressed as

$$
\tau=\mu_{1} / v_{1}+\mu_{2} / v_{2}+\ldots+\mu_{n} / v_{n}
$$

e.g,

$$
\text { True }=0.3 / 0.6+0.5 / 0.7+0.7 / 0.8+0.9 / 0.9+1 / 1
$$

As a simple illustration suppose that the meaning of true is defined by

$$
\tau_{\text {true }}(v)= \begin{cases}0 & \text { for } 0 \leq \mathrm{v} \leq \alpha \\ ((v-\alpha) /(1-\alpha))^{2} & \text { for } \alpha \leq \mathrm{v} \leq \alpha+1 / 2 \\ 1-((v-1) /(1-\alpha))^{2} & \text { for } \alpha+1 / 2 \leq \mathrm{v} \leq 1\end{cases}
$$

where $\alpha$ is a value in $[0,1]$.

Then we may write

$$
\text { true }=\int_{\alpha}^{(\alpha+1) / 2}((v-\alpha) /(1-\alpha))^{2} / v+\int_{(\alpha+1) / 2}^{1}\left[1-((v-\alpha) /(1-\alpha))^{2}\right] / v
$$

If $v_{1}=0.1, v_{2}=0.2, \ldots, v_{11}=1$

then 'true' might be written as

$$
\text { true }=0.3 / 0.7+0.5 / 0.8+0.8 / 0.9+1 / 1
$$




\subsubsection{Comparison of Linguistic Fuzzy Truth Values and Numerical Lukasiewicz Truth Values}

At the first time, it may appear that we are moving in a wrong direction, since it is certainly easier to manipulate with the real numbers in $[0,1]$. The answer is two-fold.

1. First, the Lukasiewicz truth-value set is a continuous, whereas that of fuzzy logic is a countable set. More importantly, in most applications to approximate reasoning, a small finite subset of the truth-values of fuzzy logic would in general, be sufficient because each fuzzy truth-value represents a fuzzy subset rather than a single element of $[0,1]$. Thus, we gain by trading the large number of simple Lukasiewicz truth-values for the small number of less simple truth-values of fuzzy logic- [5].

2. The second and related point is that approximate reasoning mostly deals with propositions which are fuzzy rather than precise, e.g, "weather is very warm", "he is looking very good", "cake is not very tasty" etc.

Clearly, the fuzzy truth-values of fuzzy logic are more commensurated with the fuzziness of such propositions than the numerical Lukasiewicz truth-values.

\subsubsection{Operations on Fuzzy Truth Values}

So far we have focused our attention on the structure of the truth value set of fuzzy logic. We turn next to some of the basic questions relating to the manipulation of linguistic fuzzy truth values in interval $[0,1]$.

To extend the definition of negation, conjunction, disjunction and implication in Lukasiewicz logic to those of fuzzy logic it is convenient to employ an extension principle for fuzzy sets which can be stated as follows [6].

\subsubsection{Extension Principle for Fuzzy Sets}

\section{For one variable}

Let us define a function ' $\mathrm{f}$ ' mapping from $\mathrm{X}$ to $\mathrm{Y}$

$$
\text { f: } \quad \mathrm{X} \rightarrow \mathrm{Y}
$$

where

$$
\begin{array}{lll}
\mathrm{X}=\left\{x_{i}\right\}, & x_{i} \in X, & \mathrm{i}=1,2, \ldots, \mathrm{n} \\
\mathrm{Y}=\left\{y_{j}\right\}, & y_{j} \in Y, & \mathrm{j}=1,2, \ldots, \mathrm{m}
\end{array}
$$


Let $\mathrm{A} \subset \mathrm{X}$ be a fuzzy set given by

$$
A=\left\{x_{i}, \mu_{A}\left(x_{i}\right)\right\}
$$

where $\mu_{A}(x i)$ are the membership degrees for $x_{i}, \mathrm{i}=1,2, \ldots, \mathrm{n}$ Then we can construct

$$
f(A)=\left\{f\left(x_{i}\right), \mu_{f(A)}\left(f\left(x_{i}\right)\right)\right\}=B
$$

where

$$
\mu_{f(A)}\left(f\left(x_{i}\right)\right)=\max _{f\left(x_{i}\right)} \mu_{A}\left(x_{i}\right)
$$

or we can also write $f(A)$ as

$$
f(A)=\Sigma_{x_{i} \epsilon X} \max _{f(x i)} \mu_{A}\left(x_{i}\right) / f\left(x_{i}\right)
$$

\section{Example}

Let

$$
X=\{-2,-1,0,1,2,3\}
$$

and $\mathrm{A}$ is fuzzy set in $\mathrm{X}$ given by

$$
A=0.1 /-2+0.2 /-1+0.3 / 0+0.5 / 1+0.8 / 2+1 / 3
$$

and function $\mathrm{f}$ is given by

$$
y=2 x^{2}+1
$$

then

$$
\begin{aligned}
& \mathrm{f}(\mathrm{A})=0.1 / 9+0.2 / 3+0.3 / 1+0.5 / 3+0.8 / 9+1 / 19 \\
& \mathrm{f}(\mathrm{A})=0.3 / 1+\max (0.2,0.5) / 3+\max (0.1,0.8) / 9+1 / 19 \\
& \mathrm{f}(\mathrm{A})=0.3 / 1+0.5 / 3+0.8 / 9+1 / 19
\end{aligned}
$$

\section{For two variables}

Let

$$
\begin{array}{lll}
\mathrm{X}=\left\{x_{i}\right\}, & x_{i} \in X, & \mathrm{i}=1,2, \ldots, \mathrm{n} \\
\mathrm{Y}=\left\{y_{j}\right\}, & y_{j} \in Y, & \mathrm{j}=1,2, \ldots, \mathrm{m} \\
\mathrm{Z}=\left\{z_{k}\right\}, & z_{k} \in Z, & \mathrm{k}=1,2, \ldots, \mathrm{p}
\end{array}
$$

be the universal sets. 
We introduce a two dimensional function

$$
Z=g(X, Y) \quad \text { or } \quad z_{k}=g\left(x_{i}, y_{j}\right)
$$

Now use two fuzzy sets

$$
\begin{aligned}
& A \subset X, A=\left\{x_{i} ; \mu_{A}\left(x_{i}\right)\right\} \\
& B \subset Y, B=\left\{y_{j} ; \mu_{B}\left(y_{j}\right)\right\}
\end{aligned}
$$

then

$$
\mathrm{g}(\mathrm{A}, \mathrm{B}) \subset \mathrm{Z}
$$

$\mathrm{g}(\mathrm{A}, \mathrm{B})$ in $\mathrm{Z}$ is a picture of function $\mathrm{g}$.

where

$$
g(A, B)=\sum_{\left(x_{i}, y_{j}\right) \in X \times Y} \max _{g\left(x_{i}, y_{j}\right)} \min \left(\mu_{A}\left(x_{i}\right), \mu_{B}\left(y_{j}\right)\right) / g\left(x_{i}, y_{j}\right)
$$

\section{Example}

Let $\mathrm{X}$ and $\mathrm{Y}$ be the two universal crisp sets given by

$$
\mathrm{X}=\{2,4,5\}, \text { and } \quad \mathrm{Y}=\{5,6,7\}
$$

and $\mathrm{A}, \mathrm{B}$ are two fuzzy sets $\mathrm{A} \subset X$ and $\mathrm{B} \subset Y$ respectively defined by

$$
\begin{aligned}
& \mathrm{A}=\text { 'large number in } \mathrm{X}^{\prime}=0.3 / 2+0.7 / 4+1 / 5 \\
& \mathrm{~B}=\text { 'large number in } \mathrm{Y}^{\prime}=0.6 / 5+0.9 / 6+1 / 7
\end{aligned}
$$

For $\quad \mathrm{Z} \subset X \times Y$

Let $\mathrm{g}(\mathrm{x}, \mathrm{y})=\mathrm{y}-\mathrm{x}$

then

$$
\begin{aligned}
& \mathrm{g}(\mathrm{A}, \mathrm{B})=\min (0.6,0.3) / 3+\min (0.6,0.7) / 1+\min (0.6,1) / 0+\min (0.9,0.3) / 4 \\
& +\min (0.9,0.7) / 2+(\min 0.9,1) / 1 \\
& \mathrm{~g}(\mathrm{~A}, \mathrm{~B})=\min (1,0.3) / 5+\min (1,0.7) / 3+\min (1,1) / 2 \\
& \mathrm{~g}(\mathrm{~A}, \mathrm{~B})=0.3 / 3+0.6 / 1+0.6 / 0+0.3 / 4+0.7 / 2+0.9 / 1+0.3 / 5+ \\
& \quad 0.7 / 3+1 / 2 \\
& \mathrm{~g}(\mathrm{~A}, \mathrm{~B})=0.6 / 0+\max (0.6,0.9) / 1+\max (0.7,1) / 2+\max (0.3,0.7) / 3 \\
& \quad+0.3 / 4+0.3 / 5 \\
& \mathrm{~g}(\mathrm{~A}, \mathrm{~B})=0.6 / 0+0.9 / 1+1 / 2+0.7 / 3+0.3 / 4+0.3 / 5
\end{aligned}
$$




\subsection{Use of Extension Principle for the Opera- tions on Fuzzy Truth Values}

We apply the extension principle to the definition of negation, conjunction, disjunction and implication in fuzzy logic. It is expedient to use linguistic fuzzy subsets $\tau$,

i.e, $\quad \tau:[0,1] \rightarrow[0,1]$ of truth expressions set

$\mathrm{T}=\{$ true, false,very true, very false, rather true, maybe true, bit true. etc $\}$

in which both members of set $\tau$ and their degrees are ranging from 0 to 1 .

Let $\mathrm{p}$ and $\mathrm{q}$ be propositions (statements) which have logical values described by the fuzzy sets $\tau(\mathrm{p})$ and $\tau(\mathrm{q})$ where

$$
\begin{gathered}
\tau(p)=\sum \mu_{\tau(p)}(x) / x, \quad \mathrm{x} \epsilon[0,1] \\
\tau(q)=\sum \mu_{\tau(q)}(y) / y, \quad \mathrm{y} \epsilon[0,1] .
\end{gathered}
$$

We should consider some of fuzzy truth values sets $\tau$ before proceeding further. We state

1. "false" $=1 / 0+0.9 / 0.1+0.7 / 0.2$

2. "very False" $=1 / 0+1 / 0.1+0.9 / 0.2$

3. "not False" $=0.6 / 0.6+0.7 / 0.7+0.8 / 0.8+0.9 / 0.9+1 / 1$

4. "true" $=0.7 / 0.8+0.9 / 0.9+1 / 1$

5. "very True" $=0.9 / 0.8+1 / 0.9+1 / 1$

6. "fairly True" $=1 / 0.8+0.9 / 0.9+0.8 / 1$

7. "moderately true" $=0.8 / 0.4+1 / 0.5+0.7 / 0.6$

8. "little True" $=1 / 0.3+0.9 / 0.4+0.8 / 0.5$

9. "very very False" $=1 / 0+1 / 0.1+0.9 / 0.2$ 


\subsubsection{Negation}

Negation for a certain fuzzy truth value $\tau(p)$ for statement ' $\mathrm{p}$ ' is denoted by $\neg \tau(p)$ and has the membership function given by

$$
\mu_{\neg \tau(p)}(v)=1-\mu_{\tau(p)}(v), \quad \mathrm{v} \epsilon[0,1]
$$

Here is another type of negation in which we negate the statement 'p' rather than the truth value set $\neg \tau(p)$. So the truth value on negate statement $\neg p$ is given by the membership degree function

$$
\mu_{\tau(\neg p)}(\mathrm{v})=\mu_{\tau(p)}(1-v), \quad \mathrm{v} \epsilon[0,1]
$$

\subsubsection{Example of Negation}

Let

$$
\mathrm{p}=\text { "John is looking good" }
$$

Suppose that this statement is moderately true.

$$
\tau(p)=\text { "moderately true" }=0.8 / 0.4+1 / 0.5+0.7 / 0.6
$$

so by definition

$$
\begin{aligned}
& \neg \tau(p)=\text { "not moderately true" } \\
& \neg \tau(p)=1-0.8 / 0.4+1-1 / 0.5+1-0.7 / 0.6 \\
& \neg \tau(p)=0.2 / 0.4+0 / 0.5+0.3 / 0.6
\end{aligned}
$$

Now for the second type of negation we make another calculation.

Consider the same proposition as in the previous example.

$\neg p=$ "John is not looking good"

with membership values

$$
\begin{aligned}
& \tau(\neg p)=0.8 / 1-0.4+1 / 1-0.5+0.7 / 1-0.6 \\
& \tau(\neg p)=0.8 / 0.6+1 / 0.5+0.7 / 0.4
\end{aligned}
$$

\subsubsection{Conjunction}

Conjunction between two truth fuzzy expressions $\tau(p)$ and $\tau(q)$ for two propositions $\mathrm{p}$ and $\mathrm{q}$ is given by

$$
\tau(p \wedge q)=\sum_{(x, y) \epsilon[0,1] \times[0,1]} \max _{\min (x, y)} \min \left(\mu_{\tau(p)}(x), \mu_{\tau(q)}(y)\right) / \min (x, y)
$$

with

$$
\begin{array}{ll}
\tau(p)=\left\{x, \mu_{\tau(p)}(x)\right\}, & \mathrm{x} \epsilon[0,1] \\
\tau(q)=\left\{y, \mu_{\tau(q)}(y)\right\}, & \mathrm{y} \epsilon[0,1]
\end{array}
$$




\subsubsection{Example of Conjunction}

Let us consider two fuzzy propositions

$$
\begin{aligned}
& \mathrm{p}=\text { "Clark does exercise" } \\
& \mathrm{q}=\text { "Clark is a healthy man" }
\end{aligned}
$$

with fuzzy truth values given by

$$
\begin{aligned}
& \tau(p)=\text { "little true" }=1 / 0.3+0.9 / 0.4+0.8 / 0.5 \\
& \tau(q)=\text { "moderately true" }=0.8 / 0.4+1 / 0.5+0.7 / 0.6
\end{aligned}
$$

so applying the conjunction formula, we have

$$
\begin{aligned}
& \tau(p \wedge q)=\min (1,0.8) / \min (0.3,0.4)+\min (1,1) / \min (0.3,0.5)+\min (1,0.7) / \min (0.3,0.6) \\
& +\min (0.9,0.8) / \min (0.4,0.4)+\min (0.9,1) / \min (0.4,0.5)+\min (0.9,0.7) / \min (0.4,0.6) \\
& +\min (0.8,0.8) / \min (0.5,0.4)+\min (0.8,1) / \min (0.5,0.5)+\min (0.8,0.7) / \min (0.5,0.6) \\
& \tau(p \wedge q)=0.8 / 0.3+1 / 0.3+0.7 / 0.3+0.8 / 0.4+0.9 / 0.4+0.8 / 0.4 \\
& +0.8 / 0.5+0.8 / 0.5+0.7 / 0.5 \\
& \tau(p \wedge q)=\max (0.8,1,0.7) / 0.3+\max (0.8,0.9,0.8) / 0.4+\max (0.8,0.8, \\
& 0.7) / 0.5 \\
& \tau(p \wedge q)=1 / 0.3+0.9 / 0.4+0.8 / 0.5
\end{aligned}
$$

so ("little true") AND ("moderately true") = "little true"

\subsubsection{Disjunction}

Disjunction between two truth fuzzy expressions $\tau(p)$ and $\tau(q)$ for two propositions $\mathrm{p}$ and $\mathrm{q}$ is given by

$$
\tau(p \vee q)=\sum_{(x, y) \in[0,1] \times[0,1]} \max _{\max (x, y)} \min \left(\mu_{\tau(p)}(x), \mu_{\tau(q)}(y)\right) / \max (x, y)
$$

where

$$
\mu_{\tau(p \vee q)}=\max _{\max (x, y)} \min \left(\mu_{\tau(p)}(x), \mu_{\tau(q)}(y)\right)
$$

\subsubsection{Example of Disjunction}

Apply this formula to the previous example, i.e.

$$
\begin{aligned}
& \tau(p)=\text { "little true" }=1 / 0.3+0.9 / 0.4+0.8 / 0.5 \\
& \tau(q)=\text { "moderately true" }=0.8 / 0.4+1 / 0.5+0.7 / 0.6
\end{aligned}
$$


then

$$
\begin{aligned}
& \tau(p \vee q)=\min (1,0.8) / \max (0.3,0.4)+\min (1,1) / \max (0.3,0.5)+\min (1,0.7) / \max (0.3,0.6) \\
& \quad+\min (0.9,0.8) / \max (0.3,0.6)+\min (0.9,1) / \max (0.4,0.4)+\min (0.9,0.7) / \max (0.4,0.5) \\
& \quad+\min (0.8,0.8) / \max (0.5,0.4)+\min (0.8,1) / \max (0.5,0.5)+\min (0.8,0.7) / \max (0.5,0.6) \\
& \begin{array}{l}
\tau(p \vee q)=0.8 / 0.4+1 / 0.5+0.7 / 0.6+0.8 / 0.4+0.9 / 0.5+0.7 / 0.6 \\
\quad+0.8 / 0.5+0.8 / 0.5+0.7 / 0.6
\end{array} \\
& \tau(p \vee q)=\max (0.8,0.8) / 0.4+\max (1,0.9,0.8,0.8) / 0.5+\max (0.7,0.7,0.7) / 0.6 \\
& \tau(p \vee q)=0.8 / 0.4+1 / 0.5+0.7 / 0.6=\tau(q)
\end{aligned}
$$

"little true" OR "moderately true" = "moderately true"

\subsubsection{Implication}

Implication of two fuzzy truth expressions $\tau(p)$ and $\tau(q)$ for propositions $\mathrm{p}$ and $\mathrm{q}$ is given by

$$
\tau(p \rightarrow q)=\sum_{(x, y) \in[0,1] \times[0,1]} \max _{\min (1,1-x+y)} \min \left(\mu_{\tau(p)}(x), \mu_{\tau(q)}(y)\right) / \min (1,1-x+y)
$$

with

$$
\mu_{\tau(p \rightarrow q)}(v)=\max _{\min (1,1-x+y)} \min \left(\mu_{\tau(p)}(x), \mu_{\tau(q)}(y)\right)
$$

\subsubsection{Example of Implication}

If we apply the implication operation to the data from the previous example, we will have

$$
\begin{aligned}
& \tau(p \rightarrow q)=\min (1,0.8) / \min (1,1.1)+\min (1,1) / \min (1,1.2)+\min (1,0.7) / \min (1,1.3) \\
& \quad+\min (0.9,0.8) / \min (1,1)+\min (0.9,1) / \min (1,1.1)+\min (0.9,0.7) / \min (1,1.2) \\
& \quad+\min (0.8,0.8) / \min (1,0.9)+\min (0.8,1) / \min (1,1)+\min (0.8,0.7) / \min (1,1.1) \\
& \begin{aligned}
\tau(p \rightarrow q)=0.8 / 1+1 / 1+0.7 / 1+0.8 / 1+0.9 / 1+0.7 / 1+0.8 / 0.9 \\
\quad+0.8 / 1+0.7 / 1
\end{aligned} \\
& \tau(p \rightarrow q)=\max (0.8,1,0.7,0.8,0.9,0.7,0.8,0.7) / 1+0.8 / 0.9 \\
& \tau(p \rightarrow q)=0.8 / 0.9+1 / 1
\end{aligned}
$$




\subsubsection{Equivalence}

Equivalence relation is actually minimum of two implication, i.e,

$$
\tau(p \leftrightarrow q)=\tau((p \rightarrow q) \wedge(q \rightarrow p))
$$

\subsubsection{Example of Equivalence}

$$
\begin{aligned}
\tau(q \rightarrow p) & =\sum_{(x, y) \in[0,1] \times[0,1]} \max _{\min (1,1-y+x)} \min \left(\mu_{\tau(p)}(x), \mu_{\tau(q)}(y)\right) / \min (1,1-y+x) \\
\tau(q & \rightarrow p)=\min (1,0.8) / \min (1,0.9)+\min (1,1) / \min (1,0.8)+\min (1,0.7) / \min (1,0.7) \\
& +\min (0.9,0.8) / \min (1,1)+\min (0.9,1) / \min (1,0.9)+\min (0.9,0.7) / \min (1,0.8) \\
& +\min (0.8,0.8) / \min (1,1)+\min (0.8,1) / \min (1,1)+\min (0.8,0.7) / \min (1,0.9) \\
& \\
\tau(q & \rightarrow p)=0.8 / 0.9+1 / 0.8+0.7 / 0.7+0.8 / 1+0.9 / 0.9+0.7 / 0.8 \\
& 0.8 / 1+0.8 / 1+0.7 / 0.9 \\
\tau(q & \rightarrow p)=0.7 / 0.7+1 / 0.8+0.9 / 0.9+0.8 / 1
\end{aligned}
$$

and we have

$$
\tau(p \rightarrow q)=0.8 / 0.9+1 / 1
$$

so equivalence is given by

$$
\begin{aligned}
& \tau(p \leftrightarrow q)=\tau((p \rightarrow q) \wedge(q \rightarrow p)) \\
& =\sum_{(x, y) \epsilon[0,1] \times[0,1]} \max _{\min (x, y)} \min \left(\mu_{\tau(p \rightarrow q)}(x), \mu_{\tau(q \rightarrow p)}(y)\right) / \min (x, y) \\
& \tau(p \leftrightarrow q)=\min (0.8,0.7) / \min (0.9,0.7)+\min (0.8,1) / \min (0.9,0.8)+ \\
& \min (0.8,0.9) / \min (0.9,0.9)+\min (0.8,0.8) / \min (0.9,1)+\min (1,0.7) / \min (1,0.7) \\
& +\min (1,1) / \min (1,0.8)+\min (1,0.9) / \min (1,0.9)+\min (1,0.8) / \min (1,1) \\
& \tau(p \leftrightarrow q)=0.7 / 0.7+0.8 / 0.8+0.8 / 0.9+0.8 / 1+0.7 / 0.7+1 / 0.8+ \\
& 0.9 / 0.9+0.8 / 1 \\
& \tau(p \leftrightarrow q)=0.7 / 0.7+\max (0.8,1) / 0.8+\max (0.8,0.9) / 0.9+0.8 / 1 \\
& \tau(p \leftrightarrow q)=0.7 / 0.7+1 / 0.8+0.9 / 0.9+0.8 / 1
\end{aligned}
$$




\subsection{Baldwin Approach to Fuzzy Truth Values}

It was too much difficult to define fuzzy subsets for different linguistic truth values and perform all the operations as we discussed in previous section. So Baldwin tried to formalize all these fuzzy truth values and found a very convenient way of describing them [7].

p-any statement (proposition)

$\tau(p)$-linguistic truth value as fuzzy set

Let us consider

$$
\tau(p)=\text { "true" }
$$

with the membership function

$\mu_{\text {true }}(\mathrm{v})=\mathrm{v}$

Then according to Baldwin

$$
\begin{aligned}
& \mu_{\text {false }}(\mathrm{v})=1-\mathrm{v} \\
& \mu_{\text {verytrue }}(\mathrm{v})=\left(\mu_{\text {true }}(v)\right)^{2}=v^{2} \\
& \mu_{\text {veryfalse }}(\mathrm{v})=\left(\mu_{\text {false }}(v)\right)^{2}=(1-v)^{2} \\
& \mu_{\text {rathertrue }}(\mathrm{v})=\left(\sqrt{\mu_{\text {true }}(v)}\right)=\sqrt{v} \\
& \mu_{\text {ratherfalse }}(\mathrm{v})=\left(\sqrt{\mu_{\text {false }}(v)}\right)=\sqrt{1-v} \\
& \mu_{\text {absolutelytrue }}(v)=\left\{\begin{array}{cc}
1 & v=1 \\
0 & v \epsilon[0,1)
\end{array}\right. \\
& \mu_{\text {absolutelyfalse }}(v)=\left\{\begin{array}{cc}
1 & v=0 \\
0 & v \epsilon(0,1]
\end{array}\right. \\
& \mu_{\text {undefined }}(v)=1 \quad v \epsilon[0,1]
\end{aligned}
$$

\subsubsection{Fuzzy Truth Value Sets in Baldwin Approach}

In Baldwin approach a fuzzy truth value set "true" used as a base set is given by the line $\mathrm{y}=\mathrm{x}$

$$
\begin{aligned}
& \text { "True" }=0.0 / 0+0.1 / 0.1+0.2 / 0.2+0.3 / 0.3+0.4 / 0.4+0.5 / 0.5+ \\
& \quad 0.6 / 0.6+0.7 / 0.7+0.8 / 0.8+0.9 / 0.9+1 / 1
\end{aligned}
$$

Then according to above formulas 


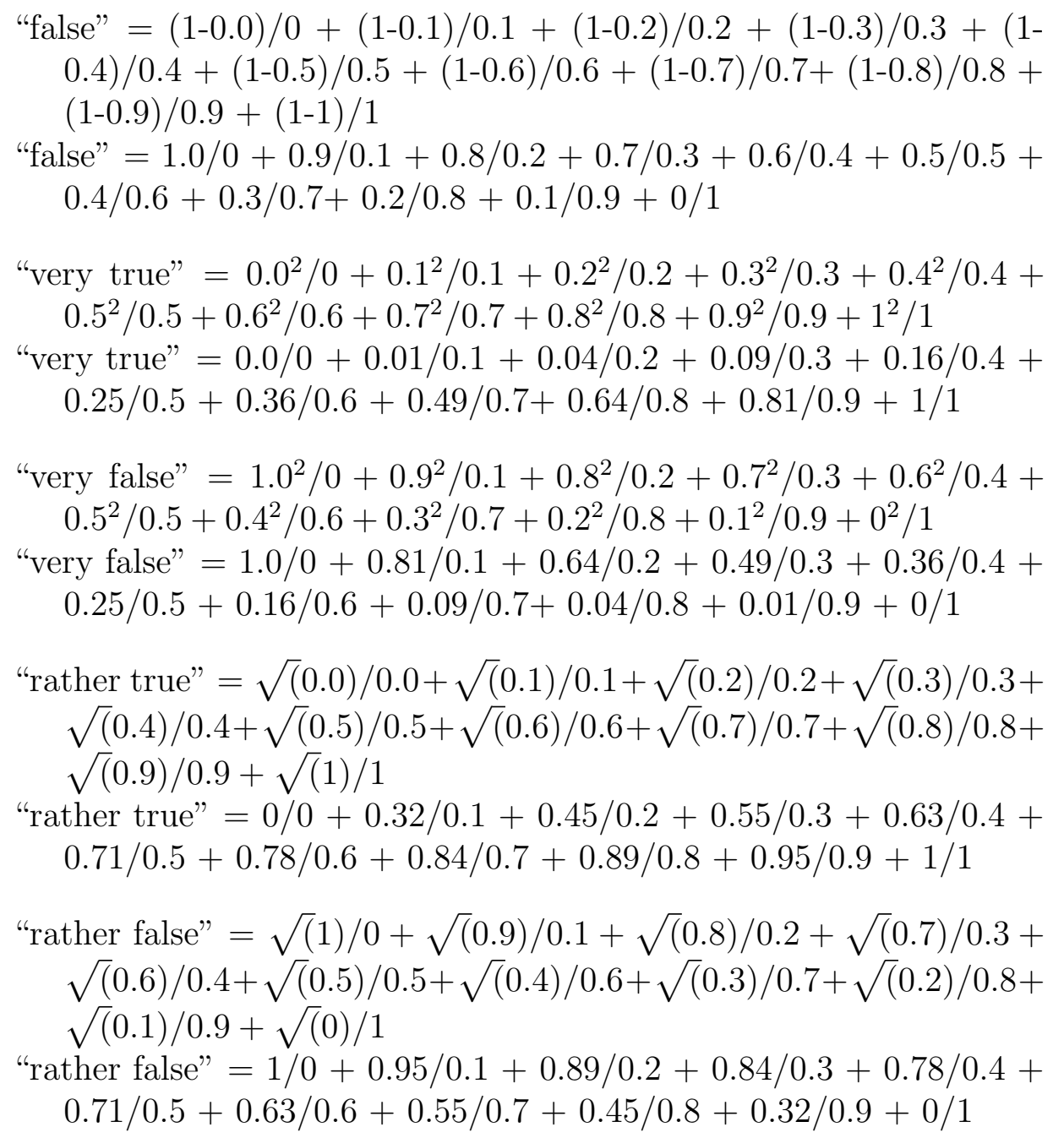

Absolute true has the value 1 for 1 and absolute false has the value 1 for 0 . They are not fuzzy sets.

\subsubsection{Negations in Baldwin Approach}

Negations of fuzzy truth values set according to Baldwin approach are given by

\begin{tabular}{ccc}
$\tau(p)$ & Negation $\neg \tau(p)$ & $\mu_{\neg \tau(p)}$ \\
\hline true & false & $1-\mu_{\text {true }}(v)=1-\mathrm{v}$ \\
very true & rather false & $1-\mu_{\text {verytrue }}(v)$ \\
& & $=1-\left(\mu_{\text {true }}(v)\right)^{2}$ \\
& & $=1-v^{2}$ \\
rather true & very false & $1-\mu_{\text {rathertrue }}(v)$ \\
& & $1-\mu_{\text {rathertrue }}(v)$ \\
& $1-\sqrt{\mu}_{\text {true }}(v)$
\end{tabular}




\subsubsection{Graphical Baldwin Approach to Fuzzy Truth Values}

Baldwin combined all the fuzzy truth values in a single graph plot shown below

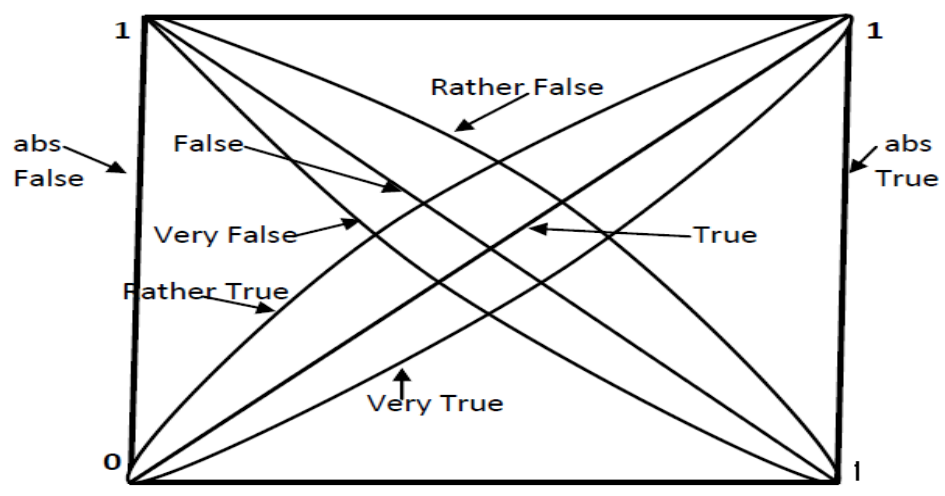

Baldwin Approach

Figure 3.2: Baldwin approach to fuzzy truth values

\subsection{Linguistic Approximation}

Due to some complication in linguistic truth-value fuzzy sets, we use linguistic approximations [8].

As we know that

$\mathrm{p}$ is a fuzzy statement with fuzzy truth value $\tau$ which can be written as

$$
p=\left\{\left(x, \mu_{p}(x)\right)\right\}, \quad x \in X
$$

with truth value

$$
\tau=\left\{\left(v, \mu_{\tau}(v)\right)\right\}, \quad v \epsilon V
$$

Here we have

$$
(\mathrm{u}=\mathrm{p})=\tau
$$

after linguistic approximation

$$
(\mathrm{u}=\mathrm{q})=\text { it is } \tau \text { that } \mathrm{p}
$$




\subsubsection{Example of Linguistic Approximation}

Let us consider the universe $\mathrm{X}$ of speed with standard units, i.e, miles/hour

$$
\mathrm{X}=\{10,20,30,40,50,60\}
$$

Let us consider the proposition $\mathrm{p}$ as

$$
\begin{aligned}
& \mathrm{p}=\text { "leopard runs fast" } \\
& \mathrm{p}=0.4 / 10+0.6 / 20+0.7 / 30+0.8 / 40+0.9 / 50+1 / 60
\end{aligned}
$$

with truth-value set

$$
\begin{aligned}
& \tau(p)=\text { "quite true" } \\
& \tau(p)=0.7 / 0.6+0.8 / 0.7+1 / 0.8+0.9 / 0.9+0.8 / 1
\end{aligned}
$$

we can combine $\mathrm{p}$ and $\tau(\mathrm{p})$ in the following sentence

$$
\mathrm{Q}=\text { "It is quite true that leopard runs fast" }
$$

given by

$$
Q=\left\{\left(x, \mu_{Q}(x)\right)\right\}
$$

where

$$
\mu_{Q}(x)=\mu_{\tau}\left(\mu_{p}(x)\right)
$$

So we get

$$
Q=0 / 10+0.7 / 20+0.8 / 30+1 / 40+0.9 / 50+0.8 / 60
$$

\subsection{Modus Ponens Law with Fuzzy truth-values}

Modus ponens law is formulated as an IF...THEN rule and was applied to classical two valued logic. This law is given by

$$
\begin{array}{ll}
\text { IF } & \mathrm{p} \rightarrow \mathrm{q} \\
\text { AND } & \mathrm{p} \\
\text { THEN } & \mathrm{q}
\end{array}
$$

or in other form

$(p \wedge(p \rightarrow q)) \rightarrow q$ which is a tautology

i.e. $\quad \tau((p \wedge(p \rightarrow q)) \rightarrow q)=$ "true" 
Here we will discuss this law for fuzzy truth values rather than classical.

We will follow the following table to prove this tautology, i.e, all values in last column must be "true".

\begin{tabular}{|c|c|c|l|l|}
\hline$\tau(p)$ & $\tau(q)$ & $\tau(p \rightarrow q)$ & $\tau(p \wedge(p \rightarrow q))$ & $\tau((p \wedge(p \rightarrow q)) \rightarrow q)$ \\
\hline true & true & & & \\
\hline true & false & & & \\
\hline false & true & & & \\
\hline false & false & & & \\
\hline
\end{tabular}

Let 'true' and 'false' be given by

$$
\begin{aligned}
& \text { "true" }=0.6 / 0.8+0.9 / 0.9+1 / 1 \\
& \text { "false" }=1 / 0+0.8 / 0.1+0.6 / 0.2
\end{aligned}
$$

1) First take fuzzy truth-values for two propositions p and $q$

$$
\begin{gathered}
\tau(p)=\text { "true" } \\
\tau(q)=\text { "true" } \\
\tau(p \rightarrow q)=\sum_{(x, y) \in[0,1] \times[0,1]} \max _{\min (1,1-x+y)} \min \left(\mu_{\tau(p)}(x), \mu_{\tau(q)}(y)\right) / \min (1,1-x+y) \\
\tau(p \rightarrow q)=\min (0.6,0.6) / \min (1,1)+\min (0.6,0.9) / \min (1,1.1)+\min (0.6,1) / \min (1,1.2) \\
+\min (0.9,0.6) / \min (1,0.9)+\min (0.9,0.9) / \min (1,1)+\min (0.9,1) / \min (1,1.1) \\
+\min (1,0.6) / \min (1,0.8)+\min (1,0.9) / \min (1,0.9)+\min (1,1) / \min (1,1) \\
\tau(p \rightarrow q)=0.6 / 1+0.6 / 1+0.6 / 1+0.6 / 0.9+0.9 / 1+0.9 / 1+ \\
0.6 / 0.8+0.9 / 0.9+1 / 1 \\
\tau(p \rightarrow q)=\max (0.6,0.9,1) / 1+\max (0.6,0.9) / 0.9+0.6 / 0.8 \\
\tau(p \rightarrow q)=0.6 / 0.8+0.9 / 0.9+1 / 1
\end{gathered}
$$

Now

$$
\begin{aligned}
& \tau(p \wedge(p \rightarrow q))=\sum_{(x, y) \in[0,1] \times[0,1]} \max _{\min (x, y)} \min \left(\mu_{\tau(p)}(x), \mu_{\tau(p \rightarrow q)}(y)\right) \\
& \tau(p \wedge(p \rightarrow q))=\min (0.6,0.6) / \min (0.8,0.8)+\min (0.6,0.9) / \min (0.8,0.9) \\
& +\min (0.6,1) / \min (0.8,1)+\min (0.9,0.6) / \min (0.9,0.8)+\min (0.9,0.9) / \min (0.9,0.9) \\
& +\min (0.9,1) / \min (0.9,1)+\min (1,0.6) / \min (1,0.8)+\min (1,0.9) / \min (1,0.9) \\
& +\min (1,1) / \min (1,1) \\
& \tau(p \wedge(p \rightarrow q))=0.6 / 0.8+0.6 / 0.8+0.6 / 0.8+0.6 / 0.8+0.9 / 0.9+ \\
& 0.9 / 0.9+0.6 / 0.8+0.9 / 0.9+1 / 1
\end{aligned}
$$




$$
\begin{aligned}
& \tau(p \wedge(p \rightarrow q))=0.6 / 0.8+0.9 / 0.9+1 / 1 \\
& \tau(p \wedge(p \rightarrow q))=\text { "true" }=\tau(p)
\end{aligned}
$$

Finally

$$
\begin{aligned}
& \tau((p \wedge(p \rightarrow q)) \rightarrow q)=\tau(p \rightarrow q) \\
& \tau((p \wedge(p \rightarrow q)) \rightarrow q)=0.6 / 0.8+0.9 / 0.9+1 / 1 \\
& \tau((p \wedge(p \rightarrow q)) \rightarrow q)=\text { "true" }
\end{aligned}
$$

Similarly, we can prove for both false truth-values i.e.

$$
\begin{aligned}
& \tau(p)=\text { "false" } \\
& \tau(q)=\text { "false" }
\end{aligned}
$$

2) If we take

$$
\begin{aligned}
& \text { "true" }=0.6 / 0.8+0.9 / 0.9+1 / 1 \\
& \text { "false" }=1 / 0+0.8 / 0.1+0.6 / 0.2
\end{aligned}
$$

Then

$$
\begin{aligned}
& \tau(p \rightarrow q)=\sum_{(x, y) \in[0,1] \times[0,1]} \max _{\min (1,1-x+y)} \min \left(\mu_{\tau(p)}(x), \mu_{\tau(q)}(y)\right) / \min (1,1-x+y) \\
& \tau(p \rightarrow q)=\min (0.6,1) / \min (1,0.2)+\min (0.6,0.8) / \min (1,0.3)+\min (0.6,0.6) / \min (1,0.4) \\
& +\min (0.9,1) / \min (1,0.1)+\min (0.9,0.8) / \min (1,0.2)+\min (0.9,0.6) / \min (1,0.3) \\
& +\min (1,1) / \min (1,0)+\min (1,0.8) / \min (1,0.1)+\min (1,0.6) / \min (1,0.2) \\
& \tau(p \rightarrow q)=0.6 / 0.2+0.6 / 0.3+0.6 / 0.4+0.9 / 0.1+0.8 / 0.2+0.6 / 0.3 \\
& +1 / 0+0.8 / 0.1+0.6 / 0.2 \\
& \tau(p \rightarrow q)=1 / 0+\max (0.8,0.9) / 0.1+\max (0.6,0.8) / 0.2+0.6 / 0.3+ \\
& 0.6 / 0.4 \\
& \tau(p \rightarrow q)=1 / 0+0.9 / 0.1+0.8 / 0.2+0.6 / 0.3+0.6 / 0.4 \\
& \tau(p \rightarrow q)=\text { "false" }
\end{aligned}
$$

Now

$$
\tau(p \wedge(p \rightarrow q))=\sum_{(x, y) \in[0,1] \times[0,1]} \max _{\min (x, y)} \min \left(\mu_{\tau(p)}(x), \mu_{\tau(p \rightarrow q)}(y)\right)
$$




$$
\begin{aligned}
& \tau(p \wedge(p \rightarrow q))=\min (0.6,1) / \min (0.8,0)+\min (0.6,0.9) / \min (0.8,0.1)+ \\
& \min (0.6,0.8) / \min (0.8,0.2)+\min (0.6,0.6) / \min (0.8,0.3)+\min (0.6,0.6) / \min (0.8,0.4) \\
& +\min (0.9,1) / \min (0.9,0)+\min (0.9,0.9) / \min (0.9,0.1)+\min (0.9,0.8) / \min (0.9,0.2) \\
& +\min (0.9,0.6) / \min (0.9,0.3)+\min (0.9,0.6) / \min (0.9,0.4)+\min (1,1) / \min (1,0) \\
& +\min (1,0.9) / \min (1,0.1)+\min (1,0.8) / \min (1,0.2)+\min (1,0.6) / \min (1,0.3) \\
& +\min (1,0.6) / \min (1,0.4) \\
& \tau(p \wedge(p \rightarrow q))=0.6 / 0+0.6 / 0.1+0.6 / 0.2+0.6 / 0.3+0.6 / 0.4+ \\
& 0.9 / 0+0.9 / 0.1+0.8 / 0.2+0.6 / 0.3+0.6 / 0.4+1 / 0+0.9 / 0.1+ \\
& 0.8 / 0.2+0.6 / 0.3+0.6 / 0.4 \\
& \tau(p \wedge(p \rightarrow q))=\max (0.6,0.9,1) / 0+\max (0.6,0.9) / 0.1+\max (0.6,0.8) / 0.2 \\
& +0.6 / 0.3+0.6 / 0.4 \\
& \tau(p \wedge(p \rightarrow q))=1 / 0+0.9 / 0.1+0.8 / 0.2+0.6 / 0.3+0.6 / 0.4 \\
& \tau(p \wedge(p \rightarrow q))=\text { "false" }
\end{aligned}
$$

Finally

$$
\begin{aligned}
& \tau((p \wedge(p \rightarrow q)) \rightarrow q)=\sum_{(x, y) \in[0,1] \times[0,1]} \max _{\min (1,1-x+y)} \min \left(\mu_{\tau(p \wedge(p \rightarrow q))}(x), \mu_{\tau(q)}(y)\right) / \min (1,1-x+y) \\
& \tau((p \wedge(p \rightarrow q)) \rightarrow q)=\min (1,1) / \min (1,1)+\min (1,0.8) / \min (1,1.1)+ \\
& \quad \min (1,0.6) / \min (1,1.2)+\min (0.9,1) / \min (1,0.9)+\min (0.9,0.8) / \min (1,1) \\
& \quad+\min (0.9,0.6) / \min (1,1.1)+\min (0.8,1) / \min (1,0.8)+\min (0.8,0.8) / \min (1,0.9) \\
& \quad+\min (0.8,0.6) / \min (1,1)+\min (0.6,1) / \min (1,0.7)+\min (0.6,0.8) / \min (1,0.8) \\
& \quad+\min (0.6,0.6) / \min (1,0.9)+\min (0.6,1) / \min (1,0.6)+\min (0.6,0.8) / \min (1,0.7) \\
& \quad+\min (0.6,0.6) / \min (1,0.8) \\
& \quad \tau((p \wedge(p \rightarrow q)) \rightarrow q)=1 / 1+0.8 / 1+0.6 / 1+0.9 / 0.9+0.8 / 1+ \\
& \quad 0.6 / 1+0.8 / 0.8+0.8 / 0.9+0.6 / 1+0.6 / 0.7+0.6 / 0.8+0.6 / 0.9 \\
& \quad+0.6 / 0.6+0.6 / 0.7+0.6 / 0.8 \\
& \tau((p \wedge(p \rightarrow q)) \rightarrow q)=\max (1,0.8,0.6) / 1+\max (0.9,0.8,0.6) / 0.9+ \\
& \quad \max (0.8,0.6,) / 0.8+0.6 / 0.7 \\
& \tau((p \wedge(p \rightarrow q)) \rightarrow q)=1 / 1+0.9 / 0.9+0.8 / 0.8+0.6 / 0.7 \\
& \tau((p \wedge(p \rightarrow q)) \rightarrow q)=\text { "true" }
\end{aligned}
$$

Similarly, we can prove for $\tau(p)=$ "false" and $\tau(q)=$ "true"

So we can concluded the following final result for fuzzy linguistic truth-values 


\begin{tabular}{|c|c|c|c|c|}
\hline$\tau(p)$ & $\tau(q)$ & $\tau(p \rightarrow q)$ & $\tau(p \wedge(p \rightarrow q))$ & $\tau((p \wedge(p \rightarrow q)) \rightarrow q)$ \\
\hline true & true & true & true & true \\
\hline true & false & false & false & true \\
\hline false & true & true & false & true \\
\hline false & false & true & false & true \\
\hline
\end{tabular}

Hence proved that $\tau((p \wedge(p \rightarrow q)) \rightarrow q)$ is a tautology for fuzzy truth values also.

Let us check this law for other fuzzy truth values.

\section{For example:}

$$
\begin{aligned}
& \tau(p)=\text { "Little True" }=1 / 0.3+0.9 / 0.4+0.8 / 0.5 \\
& \tau(q)=\text { "Very False" }=1 / 0+1 / 0.1+0.9 / 0.2 \\
& \tau(p \rightarrow q)=\sum_{(x, y) \epsilon[0,1] \times[0,1]} \max _{\min (1,1-x+y)} \min \left(\mu_{\tau(p)}(x), \mu_{\tau(q)}(y)\right) / \min (1,1-x+y) \\
& \\
& \\
& \quad+\min (0.9,1) / \min (1,0.6)+\min (0.9,1) / \min (1,0.7)+\min (0.9,0.9) / \min (1,0.5)+\min (0.8,1) / \min (1,0.6)+\min (0.8,0.9) / \min (1,0.7) \\
& \quad \\
& \quad(p \rightarrow q)=1 / 0.7+1 / 0.8+0.9 / 0.9+0.9 / 0.6+0.9 / 0.7+0.9 / 0.8+ \\
& \quad 0.8 / 0.5+0.8 / 0.6+0.8 / 0.7 \\
& \tau(p \rightarrow q)=\max (0.9,0.8) / 0.6+\max (1,0.9,0.8) / 0.7+\max (1,0.9) / 0.8 \\
& \quad+0.9 / 0.9 \\
& \tau(p \rightarrow q)=0.9 / 0.6+1 / 0.7+1 / 0.8+0.9 / 0.9
\end{aligned}
$$

Now

$$
\begin{aligned}
& \tau(p \wedge(p \rightarrow q))=\sum_{(x, y) \in[0,1] \times[0,1]} \max _{\min (x, y)} \min \left(\mu_{\tau(p)}(x), \mu_{\tau(p \rightarrow q)}(y)\right) \\
& \tau(p \wedge(p \rightarrow q))=\min (1,0.9) / \min (0.3,0.6)+\min (1,1) / \min (0.3,0.7)+ \\
& \quad \min (1,1) / \min (0.3,0.8)+\min (1,0.9) / \min (0.3,0.9)+\min (0.9,0.9) / \min (0.4,0.6) \\
& \quad+\min (0.9,1) / \min (0.4,0.7)+\min (0.9,1) / \min (0.4,0.8)+\min (0.9,0.9) / \min (0.4,0.9) \\
& \quad+\min (0.8,0.9) / \min (0.5,0.6)+\min (0.8,1) / \min (0.5,0.7)+\min (0.8,1) / \min (0.5,0.8) \\
& \quad+\min (0.8,0.9) / \min (0.5,0.9) \\
& \tau(p \wedge(p \rightarrow q))=0.9 / 0.3+1 / 0.3+1 / 0.3+0.9 / 0.3+0.9 / 0.4+0.9 / 0.4 \\
& \quad+0.9 / 0.4+0.9 / 0.4+0.8 / 0.5+0.8 / 0.5+0.8 / 0.5+0.8 / 0.5
\end{aligned}
$$




$$
\begin{aligned}
& \tau(p \wedge(p \rightarrow q))=\max (0.9,1) / 0.3+0.9 / 0.4+0.8 / 0.5 \\
& \tau(p \wedge(p \rightarrow q))=1 / 0.3+0.9 / 0.4+0.8 / 0.5=\tau(p) \\
& \tau(p \wedge(p \rightarrow q))=\tau(p)
\end{aligned}
$$

Finally

$$
\begin{aligned}
& \tau((p \wedge(p \rightarrow q)) \rightarrow q)=\tau(p \rightarrow q) \\
& \tau((p \wedge(p \rightarrow q)) \rightarrow q)=0.9 / 0.6+1 / 0.7+1 / 0.8+0.9 / 0.9 \\
& \tau((p \wedge(p \rightarrow q)) \rightarrow q)=\text { "rather true" }
\end{aligned}
$$

So Modus ponens law for fuzzy truth value expressions lead to the increasing truthfulness. 


\section{Chapter 4}

\section{Approximate Reasoning}

We have discussed fuzzy logic in previous chapter. The idea of linguistic fuzzy models imitating the human way of thinking was proposed by Zadeh in his pioneering work [3].

"The term Approximate reasoning refers to methods and methodologies that enable reasoning with imprecise inputs to obtain meaningful outputs".

Inference in approximate reasoning is in sharp contrast to inference in classical logic. Inference in approximate reasoning is computation [1] with fuzzy sets that represents the meaning of a certain set of fuzzy propositions. One of the best known application areas of fuzzy logic is approximate reasoning. Approximate reasoning with fuzzy sets encompasses a wide variety of inference schemes and have been readily applied in many fields like decision making, expert systems and fuzzy control. We can optimize the approximate judgment with the help of system of relational assignment equations based on fuzzy logic in more generalized form.

Since fuzzy logic handle approximate information in a very systematic way, it is perfect for controlling nonlinear systems and also for modeling complex systems where an inexact model exists or systems where vagueness is common. A typical fuzzy system consists of a fuzzy rule base, membership functions and an inference.

Fuzzy logic with approximate reasoning describes relations by if-then rules, such as, "if heating valve is close then temperature is low". The uncertainity in the linguistic terms (e.g. low temperature) is represented by fuzzy sets.

Each linguistic fuzzy set in this case shows the temperature range from its one extreme to the other.

The smooth outcome of reasoning with fuzzy sets is a kind of interpolation. 


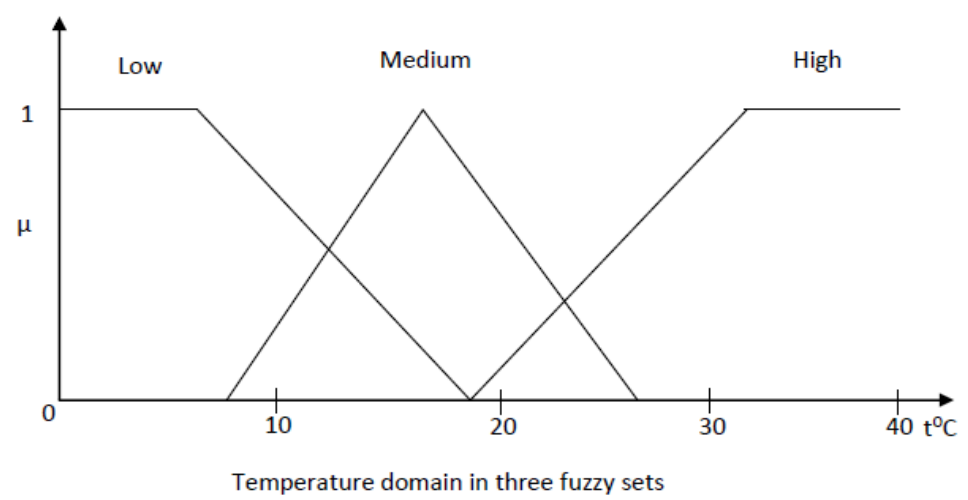

Figure 4.1: Fuzzy sets of temperature domain

\subsection{Classical IF-THEN Rule}

If-then rule in classical reasoning is given as

"If antecedent proposition Then consequent proposition."

Both propositions in this case are classical two valued.

\subsubsection{Example of Classical IF-THEN Rule}

If it is raining then take the "Umbrella"

Now if we know that it is raining, i.e, $\mathrm{A}$ is true then we deduce that $\mathrm{B}$ is true, i.e, we need to take the umbrella.

\subsection{Fuzzy IF-THEN Rule}

In fuzzy system the antecedent and consequent propositions are fuzzy sets with linguistic labels.

The linguistic model with fuzzy sets has been introduced to capture qualitative knowledge in the form of if-then rule [9].

$$
\text { If } \mathrm{x} \text { is } A_{i} \text { Then } \mathrm{y} \text { is } B_{i}, \quad \mathrm{i}=1,2, \ldots, \mathrm{k}
$$


where

$\mathrm{x}$ is a linguistic antecedent variable

$A_{i}$ are antecedent linguistic labels.

Similarly

$\mathrm{y}$ is a consequent linguistic variable.

$B_{i}$ are consequent linguistic terms.

The linguistic terms $A_{i}\left(B_{i}\right)$ are fuzzy sets.

\subsubsection{Example of Linguistic Fuzzy sets}

This example shows a linguistic variable "pressure" with three linguistic terms "high", "medium", "low". Pressure is in appropriate physical units.

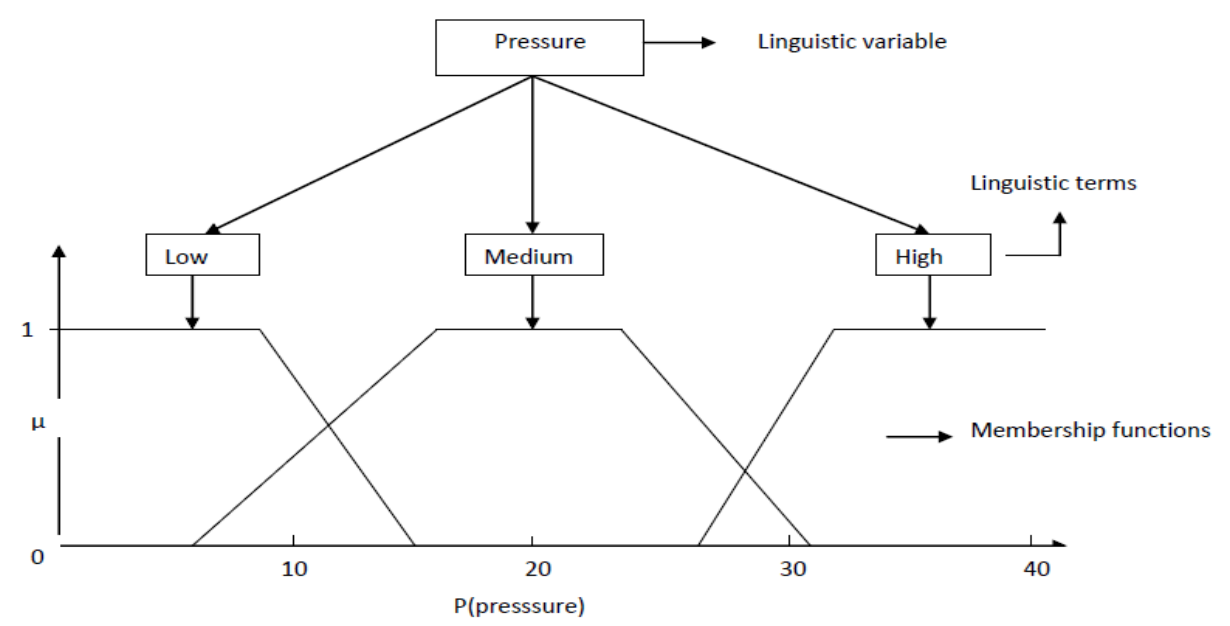

Figure 4.2: Linguistic fuzzy sets

\subsection{Models for Approximations}

1. Linguistic Fuzzy Model

In this type of the model both antecedent and consequent are fuzzy propositions. 


\section{Fuzzy Relational Model}

This model is a generalization of the linguistic model in which particular antecedent propositions are associated with several different consequent propositions through a fuzzy relation [10].

\subsection{Schematic Procedure}

Complicated propositions are difficult to approximate so in approximate reasoning we use the following scheme.
A: $\quad(\mathrm{u}=\mathrm{p}) \rightarrow(\mathrm{v}=\mathrm{q})$
$\mathrm{B}: \quad \mathrm{u}=p$
$\mathrm{C}: \quad \mathrm{v}=$ ?

So we have the implication

(A) $\mathrm{p} \rightarrow \mathrm{q}$ as if $\mathrm{u}$ is $\mathrm{p}$ then $\mathrm{v}$ is $\mathrm{q}$

(B) and $\mathrm{u}=p$

(C) what should be the answer?

We approximate the conclusion $\mathrm{v}$ using implication rule and generalized modus ponens. Example
A: $\quad(u=$ "weather is cold" $) \rightarrow(v=$ "Dress warmly" $)$
B: $\quad \mathrm{u}=$ weather is quite chilly
$\mathrm{C}: \quad \mathrm{v}=$ ?

Here we apply fuzzy rule of inference to find the approximate conclusion v. Conclusion could be of the form Dress rather warmly.

\subsection{Basic Rules of Inference}

The basic rule of inference is a traditional two valued logical reasoning and it is called Modus Ponens. If we have the implication $\mathrm{p} \rightarrow \mathrm{q}$ of two statements "p" and "q" in accordance with the present, then we can infer [11] the truth of statements based on the $q$ which authenticity "p" and the implication $p \rightarrow q$

So we conclude the following scheme.

$\begin{array}{ll}\text { If } & \mathrm{p} \rightarrow \mathrm{q} \\ \text { and } & \mathrm{p} \\ \text { Then } & \mathrm{q}\end{array}$

we can also write in this form 


$$
\mathrm{v}((\mathrm{p} \wedge(\mathrm{p} \rightarrow \mathrm{q})) \rightarrow q)=\text { "true" }
$$

\subsubsection{Example of Modus Ponens}

Let the sentences $\mathrm{p}$ and $\mathrm{q}$ are given by

$\mathrm{p}=$ "John is in Hospital"

$\mathrm{q}=$ "John is ill"

Now if this is true that John is in hospital then the statement "John is ill" is also true.

Similarly the second important rule of inference is modus tollens. It can be shown by the following scheme.

If $\quad \mathrm{p} \rightarrow \mathrm{q}$

and $\neg q$

Then $\neg p$

we can also write in this form

$$
((\neg q \wedge(p \rightarrow q)) \rightarrow \neg p)=\text { "true" }
$$

\subsubsection{Example of Modus Tollens}

We can explain the same example here also.

$\mathrm{p}=$ "John is ill".

$\mathrm{q}=$ "John is in hospital."

Now the negation on ' $\mathrm{q}$ ' is given by

$\neg q=$ "John is not in hospital"

Then we conclude according to modus tollens that

$\neg p=$ "John is not ill"

These are the cases where reasoning is exact (two valued logic) but now we extend these rules to the case of fuzzy propositions, because in nature human reasoning is approximate rather than exact. We most often deal with situations where the statements 'p' and 'q' are characterized (in the semantic sense) by some fuzzy sets. 


\subsection{Generalized Modus Ponens or Fuzzy Rule of Inference}

Let us consider $\mathrm{X}$ and $\mathrm{Y}$ as two Universal crisp sets.

Let $\mathrm{P}, \mathrm{Q}, \mathrm{S}$ be fuzzy sets created on the basis of fuzzy statements $\mathrm{p}, \mathrm{q}$ and $\mathrm{s}$ respectively with elements from universe $\mathrm{X}$ and $\mathrm{Y}$.

These fuzzy sets can be written as

$$
\begin{aligned}
& \mathrm{P}=\left(\mathrm{x}, \mu_{i} P(x)\right) \\
& \mathrm{Q}=\left(\mathrm{y}, \mu_{j} Q(y)\right) \\
& \mathrm{S}=\left(\mathrm{y}, \mu_{j} S(x)\right)
\end{aligned}
$$

where

$$
\begin{aligned}
& \mathrm{P} \subseteq X, \quad \mathrm{Q} \subseteq \mathrm{Y}, \mathrm{S} \subseteq \mathrm{Y} \\
& \text { and } P^{\prime} \subseteq \mathrm{X}, Q^{\prime} \subseteq \mathrm{Y}
\end{aligned}
$$

$\mathrm{P}$ and $\mathrm{Q}$ are fuzzy sets correspond to general statements and $P^{\prime}$ and $Q^{\prime}$ are similar to $\mathrm{P}$ and $\mathrm{Q}$ but not necessarily equal to them respectively [7]. Thus the scheme of inference is given as

$$
\begin{aligned}
& \text { If } \quad \mathrm{p}=\mathrm{P} \text { then } \mathrm{q}=\mathrm{Q} \text { Else } \mathrm{s}=\mathrm{S} \\
& \text { And } \quad p^{\prime}=P^{\prime} \\
& \text { Then } q^{\prime}=Q^{\prime} \\
& \text { where } \\
& Q^{\prime}=P^{\prime} \circ(P \rightarrow Q)
\end{aligned}
$$

We can also write this rule in these forms

If $\quad \mathrm{p}=\mathrm{P} \rightarrow(\mathrm{q}=\mathrm{Q}$ or $\mathrm{s}=\mathrm{S})$

And $\quad p^{\prime}=P^{\prime}$

Then $\quad q^{\prime}=Q^{\prime}=P^{\prime} \circ(P \rightarrow($ Qor $S))$

or

$$
\begin{array}{ll}
\text { If } & p^{\prime}=P^{\prime} \\
\text { And } & \mathrm{p}=\mathrm{P} \rightarrow(\mathrm{q}=\mathrm{Q} \text { or } \mathrm{s}=\mathrm{S}) \\
\text { Then } & q^{\prime}=Q^{\prime}=P^{\prime} \circ(P \rightarrow(\text { Qor } S))
\end{array}
$$

where

$$
\mathrm{P} \rightarrow(\mathrm{Q} \text { or } \mathrm{S})=\text { If } \mathrm{p}=\mathrm{P} \text { then } \mathrm{q}=\mathrm{Q} \text { Else } \mathrm{s}=\mathrm{S}
$$

and $P^{\prime} \subset X$

and $\mathrm{P} \rightarrow \mathrm{Q} \subset \mathrm{X} \times \mathrm{Y}$

If $\mathrm{P}$ then $\mathrm{Q}$ Else $\mathrm{S}$ is equivalent to a fuzzy relation $\mathrm{R} \subseteq X \times \mathrm{Y}$, where the various possible definitions of the fuzzy relations $\mathrm{R}$ are given by the following rules. 


\subsection{Rules of Finding Fuzzy Relation R}

There are different approaches to find out the implication fuzzy relation $\mathrm{R}$.

\subsubsection{Rule of max-min}

This rule is given by

$$
R_{m m}=(P \rightarrow Q) \vee(\neg P \rightarrow S)
$$

with the membership degrees function

$$
\mu_{R_{m m}}(x, y)=\left(\mu_{P}(x) \wedge \mu_{Q}(y)\right) \vee\left(\left(1-\mu_{P}(x)\right) \wedge \mu_{S}(y)\right)
$$

It is a term most often used.

\subsubsection{Binary Rule}

Fuzzy relation $\mathrm{R}$ in the binary rule is given by

$$
R_{b}=(\neg P \vee Q) \cap(P \vee S)
$$

with membership function

$$
\mu_{R_{b}}(x, y)=\left(\left(1-\mu_{P}(x)\right) \vee \mu_{Q}(y)\right) \wedge\left(\mu_{P}(x) \vee \mu_{S}(y)\right)
$$

\subsubsection{Lukasiewicz Rule}

Fuzzy relation $\mathrm{R}$ is given by

$$
R_{L}=(P \rightarrow Q) \cap(\neg P \rightarrow S)
$$

with membership degree function

$$
\mu_{R_{L}}(x, y)=1 \wedge\left(1-\mu_{P}(x)+\mu_{Q}(y)\right) \wedge\left(\mu_{P}(x)+\mu_{S}(y)\right)
$$

\subsubsection{Min-Rule}

In this case fuzzy relation $\mathrm{R}$ is given by

$$
R_{m}=P \times Q=P \wedge Q
$$

with membership degree fucntion

$$
\mu_{R_{m}}(x, y)=\mu_{P}(x) \wedge \mu_{Q}(y)
$$




\subsubsection{Example of Finding Fuzzy Relation R}

Let us take volume and mass in standard units, i.e, $\mathrm{m}^{3}$ and $\mathrm{kg}$ respectively given by crisp sets

$$
\begin{aligned}
& \mathrm{X}=\text { "volume" }=\{10,15,20,25\} \\
& \mathrm{Y}=\text { "mass" }=\{40,60,80,100\}
\end{aligned}
$$

Fuzzy conditional sentence may be as follows

If ( $\mathrm{x}$ is small enough) Then ( $\mathrm{y}$ is rather heavy) Else ( $\mathrm{y}$ is rather light)

$$
\begin{aligned}
& \mathrm{P}=\text { "x is small enough" }=1 / 10,0.9 / 15,0.5 / 20,0.1 / 25 \\
& \mathrm{Q}=\text { "y is rather heavy" }=0.3 / 40,0.6 / 60,0.8 / 80,1 / 100 \\
& \mathrm{~S}=\text { "y is rather light" }=1 / 40,0.7 / 60,0.5 / 80,0.2 / 100
\end{aligned}
$$

We now perform the operations discussed above to find $\mathrm{R}$ by using

1) Max-min Rule

\begin{tabular}{c|cccc} 
& 40 & 60 & 80 & 100 \\
\hline 10 & 0.9 & 0.7 & 0.5 & 0.2 \\
\hline 15 & 0.5 & 0.5 & 0.5 & 0.5 \\
\hline 20 & 0.3 & 0.6 & 0.8 & 0.9 \\
\hline 25 & 0.3 & 0.6 & 0.8 & 1.0
\end{tabular}

2) Binary Rule

\begin{tabular}{c|cccc} 
& 40 & 60 & 80 & 100 \\
\hline 10 & 0.9 & 0.7 & 0.5 & 0.2 \\
\hline 15 & 0.5 & 0.6 & 0.5 & 0.5 \\
\hline 20 & 0.3 & 0.6 & 0.8 & 0.9 \\
\hline 25 & 0.3 & 0.6 & 0.8 & 1.0
\end{tabular}

3) Lukasiewicz Rule

\begin{tabular}{l|llll} 
& 40 & 60 & 80 & 100 \\
\hline 10 & 1.0 & 0.8 & 0.6 & 0.3 \\
\hline 15 & 0.8 & 1.0 & 1.0 & 0.7 \\
\hline 20 & 0.4 & 0.7 & 0.9 & 1.0 \\
\hline 25 & 0.3 & 0.6 & 0.8 & 1.0
\end{tabular}

For any fuzzy propositions $\mathrm{P} \subseteq X, \mathrm{Q} \subseteq \mathrm{Y}, \mathrm{S} \subseteq \mathrm{Y}$, we have

$$
R_{m m} \subset R_{b} \subset R_{L}
$$


i.e.

$$
\mu_{R_{m m}}(x, y) \subseteq \mu_{R_{b}}(x, y) \subseteq \mu_{R_{L}}(x, y)
$$

\subsubsection{Example of Fuzzy Modus Ponens}

Let us consider two factors "Area" and "Amount of energy" with standard physical units, (i.e, $m^{2}$ and joule $(\mathrm{J})$ respectively) given by crisp sets

$$
\begin{aligned}
& \mathrm{X}=\text { "Area" }=\{5,20,30\} \\
& \mathrm{Y}=\text { "Amount of stored energy" }=\{100,200,300\}
\end{aligned}
$$

Now the general statement $\mathrm{P} \subseteq \mathrm{X}$ is defined as

$$
\mathrm{P}=\text { "subject is rather big" }=0.3 / 5+1 / 20+0.8 / 30
$$

The particular statement $P^{\prime}$ which is similar but not equal to $\mathrm{P}$ is given by

$$
P^{\prime}=\text { "Solar panel is big" }=0.2 / 5+0.9 / 20+1 / 30
$$

General conclusion statements are

$$
\begin{aligned}
& \mathrm{Q}=\text { "Energy production is quite high" }=0.4 / 100+0.7 / 200+1 / 500 \\
& \mathrm{~S}=\text { "Energy production is quite low" }=1 / 100+0.6 / 200+0.2 / 500
\end{aligned}
$$

The particular conclusion statement is going to be evaluated as

$$
Q^{\prime}=\text { "Energy production in solar panel" }
$$

We have the following formula

$$
Q^{\prime}=P^{\prime} \circ(P \rightarrow \mathrm{Q} \text { or } \mathrm{S})
$$

i.e.

IF "Solar panel is big" $\left(P^{\prime}\right)$

AND "the subject is rather big" (P)IMPLIES "the energy production is quite high" ELSE "the energy production is quite low"

Now we can construct the fuzzy relation $\mathrm{R} \subseteq X \times Y$ using any of the above methods.

Here we are using max-min rule to find $\mathrm{R}$ with membership degree

$$
\mu_{R_{m m}}(x, y)=\left(\mu_{P}(x) \wedge \mu_{Q}(y)\right) \vee\left(\left(1-\mu_{P}(x)\right) \wedge \mu_{S}(y)\right)
$$

so 


$$
\begin{aligned}
\mathrm{R}= & 0.3 / 5,100+0.3 / 5,200+0.3 / 5,500+0.4 / 20,100+0.7 / 20,200 \\
& +1 / 20,500+0.4 / 30,100+0.7 / 30,200+0.8 / 30,500+0.7 / 5,100 \\
& +0.6 / 5,200+0.2 / 5,500+0 / 20,100+0 / 20,200+0 / 20,500+ \\
& 0.2 / 30,100+0.2 / 30,200+0.2 / 30,500 \\
\mathrm{R}= & 0.7 / 5,100+0.6 / 5,200+0.3 / 5,500+0.4 / 20,100+0.7 / 20,200+ \\
& 1 / 20,500+0.4 / 30,100+0.7 / 30,200+0.8 / 30,500
\end{aligned}
$$

In matrix form

$$
\mu_{R_{m m}}(x, y)=\left(\begin{array}{ccc}
0.7 & 0.6 & 0.3 \\
0.4 & 0.7 & 1.0 \\
0.4 & 0.7 & 0.8
\end{array}\right)
$$

Now if we want to obtain the final result

i.e. $Q^{\prime}=$ "Energy production in solar panel"

then we have to perform the max-min operation of the composition

$$
\begin{gathered}
Q^{\prime}=\left[\begin{array}{lll}
0.2 & 0.9 & 1.0
\end{array}\right] \circ\left[\begin{array}{lll}
0.7 & 0.6 & 0.3 \\
0.4 & 0.7 & 1.0 \\
0.4 & 0.7 & 0.8
\end{array}\right] \\
Q^{\prime}=\left[\begin{array}{lll}
0.4 & 0.7 & 0.9
\end{array}\right]
\end{gathered}
$$

to reveal the fuzzy set

$$
Q^{\prime}=0.4 / 100+0.7 / 200+0.9 / 500 .
$$

We will choose the value with highest membership degree. In this case 500 has the maximum membership degree 0.9 , so the approximation of energy production in given situation is 500 .

\subsubsection{Conjunction Form of the Antecedent}

Extended form of antecedent is in conjunction form [12], generally given by

$$
\begin{aligned}
& R_{i} \text { : If } p_{1} \text { is } A_{i 1} \text { and } p_{2} \text { is } A_{i 2} \text { and, } \ldots, p_{p} \text { is } A_{i p} \text { Then y is } B_{i} \\
& \mathrm{i}=1,2, \ldots . \mathrm{k}
\end{aligned}
$$

A single conjunction of $A_{i}$ is the cartesian product conjunction of fuzzy sets $A_{i j}$ with $\mathrm{j}=1,2, \ldots, \mathrm{p}$

$$
A_{i}=A_{i 1} \times A_{i 2} \times \ldots \ldots \ldots A_{i p}
$$

with the membership function 


$$
\mu_{A_{i}}\left(x_{1}, x_{2}, \ldots, x_{p}\right)=\mu_{A_{i 1}}\left(x_{1}\right) \wedge \mu_{A_{i 2}}\left(x_{2}\right) \wedge \ldots \wedge \mu_{A_{i p}}\left(x_{p}\right)
$$

where

$$
\begin{aligned}
& x_{1} \epsilon X_{1}, x_{2} \epsilon X_{2}, \ldots, x_{p} \epsilon X_{p} \\
& X_{1}, X_{2}, \ldots, X_{p} \text { are universes. }
\end{aligned}
$$

\section{Example}

Let us consider a simple antecedent with only two conjunctions statements. First we define the universes

$$
\begin{aligned}
& X_{1}=\text { "temperature" }=\{20,40,60\} \\
& X_{2}=\text { "pressure" }=\{5,10,15\}
\end{aligned}
$$

where temperature and pressure are in standard physical units, i.e, centigrade and Pascal respectively.

Now if the antecedent is of the form

If the temperature is moderate and pressure is high

where fuzzy sets are

$$
\begin{aligned}
& A_{1}=\text { "temperature is moderate" }=0.3 / 20+1 / 40+0.4 / 60 \\
& A_{2}=\text { "pressure is high" }=0.1 / 5+0.6 / 10+1 / 15
\end{aligned}
$$

Now single conjunction $\mathrm{A}$ is given by

$$
\mathrm{A}=\text { "temperature is moderate and pressure is high" }
$$

$$
\begin{aligned}
\mathrm{A}= & \min (0.3,0.1) / 20,5+\min (0.3,0.6) / 20,10+\min (0.3,1) / 20,15+ \\
& \min (1,0.1) / 40,5+\min (1,0.6) / 40,10+\min (1,1) / 40,15+\min (0.4,0.1) / 60,5 \\
& +\min (0.4,0.6) / 60,10+\min (0.4,1) / 60,15 \\
& \\
\mathrm{~A}= & 0.1 / 20,5+0.3 / 20,10+0.3 / 20,15+0.1 / 40,5+0.6 / 40,10+ \\
& 1 / 40,15+0.1 / 60,5+0.4 / 60,10+0.4 / 60,15
\end{aligned}
$$

$$
A=\begin{array}{ccc}
5 & 10 & 15 \\
20 \\
40 \\
60
\end{array}\left[\begin{array}{lll}
0.1 & 0.3 & 0.3 \\
0.1 & 0.6 & 1.0 \\
0.1 & 0.4 & 0.4
\end{array}\right]
$$





\section{Chapter 5}

\section{Approximate Reasoning in Chemical Reactions}

Chemical reactions occur at different rates. These reaction rates depend upon some key factors. We use fuzzy logic and approximate reasoning in the evaluation of rates at which the chemical reaction is performed, where chemical reactions are characterized in terms of multiple factors. The method is illustrated by using imprecise information (conditional propositions and implications). Propositions involve factors on which reactions rate depend, and consequent is the measure of reactions rate associated with these factors.

Some methods for the evaluation of reaction rates have appeared in recent years. Here we use approximate reasoning which cover uncertainty and imprecision characteristics of evaluation. Impression is non-random rather than statistical nature. We use expressions with linguistic variables as fuzzy sets in the evaluation.

Chemical reaction some times goes out of the control which can create a serious problem with the risk of damage to property and injury to people. During the manufacture, raw material react together and give the product. Such exothermic reaction release energy. Some times with the change of surrounding environment, the reaction rate change and we need to be very careful while performing reactions. Using fuzzy logic and approximate reasoning, we will evaluate a reaction rate in the given environment and other sensitive conditions.

There are two key factors on which reaction rates depend. These factors are

1. Temperature

2. Particle size

Basically the reaction rate depends upon the frequency of the collisions between particles. If the collision is frequent than fast will be the rate and vice versa. And 
frequency of collision increases with increasing temperature and particle size.

Each chemical reaction has both maximum and minimum range of rate. And the variations from the boundaries can create serious problems. Below the minimum rate, the reaction will not occur and above the maximum value it is very dangerous.

Let us consider the values of temperature and particle sizes for a chemical reaction. The universal crisp set $X_{1}$ is given by

$X_{1}$ which defines temperature states-levels $X_{1}=\left\{T_{1}, T_{2}, T_{3}\right\}$

These levels cover the whole range of $X_{1}$ from minimum level $T_{1}$ to maximum level $T_{3}$.

Another universe $X_{2}$ defines particle sizes

$X_{2}=\left\{D_{1}, D_{2}, D_{3}, D_{4}\right\}$

These sizes are arranged in ascending order, i.e, $D_{1}$ corresponds to the minimum and $D_{4}$ assist maximum size.

In universe $\mathrm{Y}$, we have the different states of reaction rate. These are the possible states of reaction rate for above levels of temperature and particle sizes.

In $\mathrm{Y}$ we define states of reaction rate as

$\mathrm{Y}=\left\{F_{0}, F_{1}, F_{2}, F_{3}, F_{4}\right\}$

These states are also stated in ascending order because reaction rate is directly proportional to the increasing value of temperature and particle size.

After defining the universes, we define a general situation in chemical reaction. After performing the experiments again and again scientists found many formulas which show that the increasing values of temperature and particle size bring the increasing reaction rate because they effect directly to the number of collisions per second which then leads to the increased reaction rate. So we are going to apply approximate reasoning of the previously discussed types to chemical reactions.

The general invariant situation [13] is

"If temperature is high and particles size is very large, then reaction rate will be maximum."

We convert this general statement to relation $\mathrm{R}$ after determining fuzzy sets for above propositions.

Compare the general statement with implication 
If $\mathrm{p}$ then $\mathrm{q}$

Then we get

$\mathrm{p}=$ "temperature is high and particles size is very large"

$\mathrm{q}=$ "reaction rate is maximum"

Here the proposition $\mathrm{p}$ is the conjunction of two propositions. Let denote them by $p_{1}$ and $p_{2}$, i.e,

$$
\begin{aligned}
& \mathrm{p}=p_{1} \text { and } p_{2} \text { or } \\
& \mathrm{p}=p_{1} \wedge p_{2}
\end{aligned}
$$

with

$$
p_{1}=\text { "temperature is high" }
$$

given by fuzzy set $P_{1}$ as

$$
P_{1}=0.3 / T_{1}+0.7 / T_{2}+1 / T_{3}
$$

where $P_{1} \subset X_{1}$

and

$$
p_{2}=\text { "particle size is very large" }
$$

given by the fuzzy set $P_{2}$ as

$$
\begin{aligned}
& P_{2}=0 / D_{1}+0.2 / D_{2}+0.6 / D_{3}+1 / D_{4} \\
& \text { where } P_{2} \subset X_{2}
\end{aligned}
$$

and now proposition ' $\mathrm{q}$ ' is given by the fuzzy set ' $\mathrm{Q}$ ' as

$$
\mathrm{Q}=0 / F_{0}+0.1 / F_{1}+0.5 / F_{2}+0.8 / F_{3}+1 / F_{4}
$$

where $\mathrm{Q} \subset \mathrm{Y}$

Now we will find the conjunction $\mathrm{P}=P_{1} \wedge P_{2}$ with membership degree function

$$
\mu_{P}\left(T_{i}, D_{j}\right)=\min \left(\mu_{P_{1}}\left(T_{i}\right), \mu_{P_{2}}\left(D_{j}\right)\right)
$$

where $\mathrm{i}=1,2,3$

and $\mathrm{j}=1,2,3,4$

so

$$
\begin{aligned}
\mathrm{P}= & P_{1} \wedge P_{2} \\
\mathrm{P}= & \mu_{P}\left(T_{1}, D_{1}\right) /\left(T_{1}, D_{1}\right)+\mu_{P}\left(T_{1}, D_{2}\right) /\left(T_{1}, D_{2}\right)+\mu_{P}\left(T_{1}, D_{3}\right) /\left(T_{1}, D_{3}\right)+ \\
& \mu_{P}\left(T_{1}, D_{4}\right) /\left(T_{1}, D_{4}\right)+\mu_{P}\left(T_{2}, D_{1}\right) /\left(T_{2}, D_{1}\right)+\mu_{P}\left(T_{2}, D_{2}\right) /\left(T_{2}, D_{2}\right)+ \\
& \mu_{P}\left(T_{2}, D_{3}\right) /\left(T_{2}, D_{3}\right)+\mu_{P}\left(T_{2}, D_{4}\right) /\left(T_{2}, D_{4}\right)+\mu_{P}\left(T_{3}, D_{1}\right) /\left(T_{3}, D_{1}\right)+ \\
& \mu_{P}\left(T_{3}, D_{2}\right) /\left(T_{3}, D_{2}\right)+\mu_{P}\left(T_{3}, D_{3}\right) /\left(T_{3}, D_{3}\right)+\mu_{P}\left(T_{3}, D_{4}\right) /\left(T_{3}, D_{4}\right)
\end{aligned}
$$


so we get

$$
\begin{aligned}
\mathrm{P}= & 0 /\left(T_{1}, D_{1}\right)+0.2 /\left(T_{1}, D_{2}\right)+0.3 /\left(T_{1}, D_{3}\right)+0.3 /\left(T_{1}, D_{4}\right)+0 /\left(T_{2}, D_{1}\right)+ \\
& 0.2 /\left(T_{2}, D_{2}\right)+0.6 /\left(T_{2}, D_{3}\right)+0.7 /\left(T_{2}, D_{4}\right)+0 /\left(T_{3}, D_{1}\right)+0.2 /\left(T_{3}, D_{2}\right)+ \\
& 0.6 /\left(T_{3}, D_{3}\right)+1 /\left(T_{3}, D_{4}\right)
\end{aligned}
$$

In tabular form

\begin{tabular}{ccccc}
$X_{1}$ & $X_{2}$ & $\mu_{P_{1}}\left(T_{i}\right)$ & $\mu_{P_{2}}\left(D_{j}\right)$ & $\mu_{P}\left(T_{i}, D_{j}\right)$ \\
\hline$T_{1}$ & $D_{1}$ & 0.3 & 0.0 & 0.0 \\
$T_{1}$ & $D_{2}$ & 0.3 & 0.2 & 0.2 \\
$T_{1}$ & $D_{3}$ & 0.3 & 0.6 & 0.3 \\
$T_{1}$ & $D_{4}$ & 0.3 & 1.0 & 0.3 \\
$T_{2}$ & $D_{1}$ & 0.7 & 0.0 & 0.0 \\
$T_{2}$ & $D_{2}$ & 0.7 & 0.2 & 0.2 \\
$T_{2}$ & $D_{3}$ & 0.7 & 0.6 & 0.6 \\
$T_{2}$ & $D_{4}$ & 0.7 & 1.0 & 0.7 \\
$T_{3}$ & $D_{1}$ & 1.0 & 0.0 & 0.0 \\
$T_{3}$ & $D_{2}$ & 1.0 & 0.2 & 0.2 \\
$T_{3}$ & $D_{3}$ & 1.0 & 0.6 & 0.6 \\
$T_{3}$ & $D_{4}$ & 1.0 & 1.0 & 1.0
\end{tabular}

Now the implication $P \rightarrow Q$ is given by fuzzy relation $\mathrm{R}$. We will find $\mathrm{R}$ using Lukasiewicz rule with membership degrees function

$$
\mu_{R}\left[\left(T_{i}, D_{j}\right), F_{k}\right]=1 \wedge\left(\left(1-\mu_{P}\left(T_{i}, D_{j}\right)\right)+\mu_{Q}\left(F_{k}\right)\right) \wedge\left(\mu_{P}\left(T_{i}, D_{j}\right)+\left(1-\mu_{Q}\left(F_{k}\right)\right)\right)
$$

where $\mathrm{i}=1,2,3 \quad \mathrm{j}=1,2,3,4 \quad \mathrm{k}=0,1,2,3,4$

So calculated $\mathrm{R}$ in matrix form is

$$
\begin{aligned}
& \begin{array}{lllll}
F_{0} & F_{1} & F_{2} & F_{3} & F_{4}
\end{array} \\
& \left(T_{1}, D_{1}\right) \quad\left[\begin{array}{lllll}
1.0 & 0.9 & 0.5 & 0.2 & 0.0
\end{array}\right. \\
& \begin{array}{l|lllll}
\left(T_{1}, D_{2}\right) & 0.8 & 0.9 & 0.7 & 0.4 & 0.2
\end{array} \\
& \begin{array}{llllll}
\left(T_{1}, D_{3}\right) & 0.7 & 0.8 & 0.8 & 0.5 & 0.3
\end{array} \\
& \begin{array}{llllll}
\left(T_{1}, D_{4}\right) & 0.7 & 0.8 & 0.8 & 0.5 & 0.3
\end{array} \\
& \begin{array}{l|lllll}
\left(T_{2}, D_{1}\right) & 1.0 & 0.9 & 0.5 & 0.2 & 0.0
\end{array} \\
& R=\begin{array}{l|lllll}
\left(T_{2}, D_{2}\right) & 0.8 & 0.9 & 0.7 & 0.4 & 0.2 \\
\left(T_{2}, D_{3}\right) & 0.4 & 0.5 & 0.9 & 0.8 & 0.6
\end{array} \\
& \begin{array}{llllll}
\left(T_{2}, D_{4}\right) & 0.3 & 0.4 & 0.8 & 0.9 & 0.7
\end{array} \\
& \begin{array}{llllll}
\left(T_{3}, D_{1}\right) & 1.0 & 0.9 & 0.5 & 0.2 & 0.0
\end{array} \\
& \begin{array}{lllllll}
\left(T_{3}, D_{2}\right) & 0.8 & 0.9 & 0.7 & 0.4 & 0.2
\end{array} \\
& \begin{array}{l|lllll}
\left(T_{3}, D_{3}\right) & 0.4 & 0.5 & 0.9 & 0.8 & 0.6
\end{array} \\
& \left(T_{3}, D_{4}\right) \quad\left[\begin{array}{lllll}
0.0 & 0.1 & 0.5 & 0.8 & 1.0
\end{array}\right]
\end{aligned}
$$


This is the relation which actually describes the implication of general situation concerning a chemical reaction.

Now we can check the reaction rate for any other values of temperature and particle size using this relation $\mathrm{R}$ and extended modus ponens rule.

Some times we use smaller size of particles according to availability. So in that cases we should variate the temperature accordingly to sustain the reaction rate as required.

So take a certain situation in this chemical reaction that we have large particles size and moderate temperature. We denote this statement with $p^{\prime}$ given by

$$
p^{\prime}=\text { "temperature is moderate and particle size is large" }
$$

This statement is again a conjunction of two propositions given by

$$
p^{\prime}=p_{1}^{\prime} \wedge p_{2}^{\prime}
$$

where

$$
p_{1}^{\prime}=\text { "temperature is moderate" }
$$

with fuzzy set

$$
P_{1}^{\prime}=0.6 / T_{1}+0.9 / T_{2}+0.4 / T_{3}
$$

and

$$
p_{2}^{\prime}=\text { "particles size is large" }
$$

with fuzzy set

$$
P_{2}^{\prime}=0.1 / D_{1}+0.5 / D_{2}+1 / D_{3}+0.7 / D_{4}
$$

So $P^{\prime}$ is given by the membership function given by equation 1 . we get

$$
\begin{aligned}
P^{\prime}= & 0.1 /\left(T_{1}, D_{1}\right)+0.5 /\left(T_{1}, D_{2}\right)+0.6 /\left(T_{1}, D_{3}\right)+0.6 /\left(T_{1}, D_{4}\right)+1 /\left(T_{2}, D_{1}\right)+ \\
& 0.5 /\left(T_{2}, D_{2}\right)+0.9 /\left(T_{2}, D_{3}\right)+0.7 /\left(T_{2}, D_{4}\right)+1 /\left(T_{3}, D_{1}\right)+0.4 /\left(T_{3}, D_{2}\right)+ \\
& 0.4 /\left(T_{3}, D_{3}\right)+0.4 /\left(T_{3}, D_{4}\right)
\end{aligned}
$$

Now use extended modus ponens rule to find the conclusion $Q^{\prime}$, where extended modus ponens is given by [3]

$\begin{array}{ll}\text { If } & P^{\prime} \\ \text { and } & P \rightarrow Q \quad(\mathrm{R}) \\ \text { Then } & Q^{\prime}=P^{\prime} \circ R\end{array}$ 
In our case

If $\quad P_{1}^{\prime} \wedge P_{2}^{\prime}$

and $\quad\left(P_{1} \wedge P_{2}\right) \rightarrow Q$

Then $\quad Q^{\prime}=\left(P_{1}^{\prime} \wedge P_{2}^{\prime}\right) \circ\left(\left(P_{1} \wedge P_{2}\right) \rightarrow Q\right)$

Now membership degree function in max-min operation $P^{\prime} \circ R$ is given by

$$
\mu_{Q^{\prime}}\left(F_{k}\right)=\operatorname{maxmin}\left(\mu_{P^{\prime}}\left(T_{i}, D_{j}\right), \mu_{R}\left(\left(T_{i}, D_{j}\right), F_{k}\right)\right)
$$

SO

$Q^{\prime}=\left[\begin{array}{llllllllllll}0.1 & 0.5 & 0.6 & 0.6 & 0.1 & 0.5 & 0.9 & 0.7 & 0.1 & 0.4 & 0.4 & 0.4\end{array}\right] \circ\left[\begin{array}{ccccc}1.0 & 0.9 & 0.5 & 0.2 & 0.0 \\ 0.8 & 0.9 & 0.7 & 0.4 & 0.2 \\ 0.7 & 0.8 & 0.8 & 0.5 & 0.3 \\ 0.7 & 0.8 & 0.8 & 0.5 & 0.3 \\ 1.0 & 0.9 & 0.5 & 0.2 & 0.0 \\ 0.8 & 0.9 & 0.7 & 0.4 & 0.2 \\ 0.4 & 0.5 & 0.9 & 0.8 & 0.6 \\ 0.3 & 0.4 & 0.8 & 0.9 & 0.7 \\ 1.0 & 0.9 & 0.5 & 0.2 & 0.0 \\ 0.8 & 0.9 & 0.7 & 0.4 & 0.2 \\ 0.4 & 0.5 & 0.9 & 0.8 & 0.6 \\ 0.0 & 0.1 & 0.5 & 0.8 & 1.0\end{array}\right]$

$$
Q^{\prime}=\left[\begin{array}{lllll}
0.6 & 0.6 & 0.9 & 0.8 & 0.7
\end{array}\right]
$$

so in fuzzy set form

$$
Q^{\prime}=0.6 / F_{0}+0.6 / F_{1}+0.9 / F_{2}+0.8 / F_{3}+0.7 / F_{4}
$$

Finally we choose the value of reaction rate with maximum membership degree i.e, $F_{2}$ with 0.9 .

This shows that the reaction rate has the value around $F_{2}$.

This method can be applied to several chemical reactions in which variation in temperature and particles size can create serious problems. So by using this method one can find the approximate value of reaction rate.

This is our idea towards the approximation of reaction rate with only two factors and small number of levels. We can also extend this idea for more factors and levels. It can be done by connecting the factors with AND and OR operations. i.e. If $\mathrm{P} 1=\mathrm{u}$ and $\mathrm{P} 2=\mathrm{v}$ or $\mathrm{P} 3=\mathrm{w}$ then $\mathrm{Q}=\mathrm{z}$ 


\section{Chapter 6}

\section{News}

Fuzzy logic was first proposed by Lotfi A. Zadeh of the University of California at Berkeley United States in a 1965. He elaborated on his ideas in a 1973 paper that introduced the concept of linguistic variables. Hence it should be possible to improve the performance of electromechanical controllers by modeling the way of handling this type of information. The theory developed slowly at first, but by the early 1970's it had attracted a small international group of scientists. This included a number of westerners, mostly mathematicians, and a small number of Japanese engineers.

Fuzzy logic has been implemented in many applications including household appliance, consumer-electronic goods, transit systems, automobiles, and industrial processes. Many consumer products using fuzzy technology are currently available in Japan, and some are now being marketed in the US and Europe. Other applications that have been reported include image analysis incorporating various human inputs, as in theories and research in it [14].

Although US was the first to introduce fuzzy Logic in different areas of practical applications but it is Japan where a leadership was taken in widely implementing the associated technology in the fields mentioned above. They use this approach in problems that involve knowledge-based decision making. So we can say that Japan benefited the global community, industry, academia, and various professions. Approximation is viewed as a capability of an "intelligent" system. Fuzzy logic is considered as one such approach of approximate reasoning Nikkei Industrial News in Japan reported in October 1987 that Toshiba Co. had developed a fuzzy logic system for controlling machinery, with the intention of applying it in industrial products, traffic control, and nuclear energy.

Embedded systems play an important role in our daily life. They have gained importance for productivity and comfort. Industrial control systems, medical instruments, transportation vehicles [15], bank machines, washing machines, vac- 
uum cleaners and many other machines now depend on embedded systems. It appears as if our current civilization is built around embedded systems. Increased market demands require embedded systems to be developed even more rapidly than before. Fuzzy Logic and neural approaches can provide a mechanism of making them more efficient.

Development of the EMERGE system was begun in 1980. Since that time, EMERGE has undergone a number of transformations. Originally, it was a knowledge-based system utilizing an extended form of production rules that allowed disjunctions, conjunctions, and counts EMERGE has been modified to use approximate reasoning techniques instead of Boolean logic. Computer-assisted medical decision support systems have been shown to be useful in a number of medical applications. One of the important theoretical advances in these systems has been the incorporation of methods for dealing with uncertain and imprecise information. Many techniques including fuzzy logic, belief networks, Bayesian systems, and approximate reasoning are being implemented in the surgical decisions.

\subsection{Latest News}

For the first time that scientists have applied fuzzy logic modeling to the field of aging. The process of aging disturbs a broad range of cellular mechanisms in a complex fashion and is not well understood. Cellular biodynamics in aging is a complex control system. Scientists have found a new approach to evaluate a person's risk of cardiovascular disease, stroke, high blood pressure, or heart failure.

Signal scattering will always be only approximations to the (time-varying) true situations. Consequently, the interpretation of the processing results is difficult and relies usually on some kind of human knowledge. Fuzzy logic has helped overcome similar decision-making difficulties in other disciplines, most notably in systems control like GPS.

The technique uses fuzzy logic to teach a neural network computer program to analyze patient data and spot correlations that can be translated into a risk factor for an individual.

Fuzzy logic based electronic control systems such as automatic transmissions, engine control and Anti-lock Brake Systems (ABS) is being used now in the automotive industry. Intel Corporation is the leading supplier of microcontrollers for ABS and enjoys a technology agreement with Inform Software Corporation the leading supplier of Fuzzy Logic tools and systems. 
The new system is one of the first to apply fuzzy logic to audio, assigning rules to sound wave data and providing a processor with instructions so that it can prioritize information. Software Called MultEQ developed by Chris Kyriakakis is based upon fuzzy logic. 



\section{Chapter 7}

\section{Conclusion}

Multi-valued logic is strictly related with fuzzy set theory and fuzzy logic. An approach to approximate reasoning in chemical reactions based upon fuzzy logic has been described in this thesis. The method is very simple and efficient based upon extended modus ponens which allows to take multiple compound statements using AND and OR operations. But we have not considered many intersections as well as significant issues. It can be applied to different chemical, fission and fusion reactions including more intersections and unions of antecedent proportions to get better result. Applications of the method can be made to such areas as pattern recognition, medical diagnosis, Artificial Intelligence and fuzzy control. The importance of such a concept would seem to be important and much research is needed in the future. 



\section{Bibliography}

[1] S. W.H., Logic an introductory course., Amsterdam:North- Holland, 1983.

[2] R. N., "A survery of many-valued logic," in Many-valued logic, Mar. 1969.

[3] Z. L.A., "Fuzzy logic and approximate reasoning." University of California Berkeley, 1975.

[4] H. J.Zimmermann, Fuzzy Set Theory and its Applications, 4th ed. london: Kluwer Academic, 1976.

[5] M. P.N., "Fuzzy logic tech memo 66-3344-1." Bell telephone lab USA, 1969.

[6] G. J. and M. Gupta., "Fuzzy sets and the social nature of truth," in Advances in fuzzy set theory and applications., Amsterdam North Holland, 1979.

[7] B. J.F., "A new approach to approximate reasoning using a fuzzy logic." vol. 2, 1979., pp. 309-325.

[8] K. G.J, "Some issues of linguistic approximation," vol. 1, IEEE conference, 2004 .

[9] Y. R.R, "Linguistic models and fuzzy truths int. j," in Man Machine studies, vol. 10, 1978, pp. 483-494.

[10] G. J. K. B. Yuan., "selected papers of lofti a. zadeh," in Fuzzy sets, fuzzy logic and fuzzy systems, World scientific publishing, 1996.

[11] D. Schwartz and G. L. Klir, "Applications of fuzzy sets and approximate reasoning," in IEEE conference, 1994.

[12] M. Mas. and T. .J., "A survey on fuzzy implication functions," IEEE Journal, 2007.

[13] C. . de Silva, "Application of fuzzy logic and approximate reasoning in process automation," in IEEE conference, 1997.

[14] chau and shun, "Forecasting exchange rates with fuzzy logic and approximate reasoning," in IEEE conference, 2000. 
[15] P.N.Smith, Application approximate reasoning in transport project evaluation. Department of geographical sciences and planning. The university of Queensland st.Lucia, Queensland., Australia, 1999. 